\title{
ANTIHOLOMORPHIC INVOLUTIONS OF ANALYTIC FAMILIES OF ABELIAN VARIETIES
}

\author{
BY
}

ALLAN ADLER

\begin{abstract}
ABSTRACr. In this paper, we investigate antiholomorphic involutions of Kuga-Satake analytic families of polarized abelian varieties $V$. A complete set of invariants of the Aut $(V)$-conjugacy classes of antiholomorphic involutions of $V$ is obtained. These invariants are expressed as cohomological invariants of the arithmetic data defining $V$. In the last section, the fibre varieties of Kuga-Satake type belonging to totally indefinite quaternion division algebras over totally real fields are investigated in more detail, and the cohomological invariants are related to results of Steve Kudla. The group of holomorphic sections of $V$ is computed for this case. It is also shown that in general the fibre structure of $V$ is intrinsic.
\end{abstract}

Introduction. In this paper, we investigate antiholomorphic involutions of certain analytic families of abelian varieties parametrized by compact local Hermitian symmetric spaces. One first observes that such an involution must be fibre preserving and therefore induce an antiholomorphic involution of the parameter space, which is an invariant of the original involution. By means of this observation, one can reduce the problem to that of describing antiholomorphic maps between complex tori, which is easily solved. Namely, every antiholomorphic map between complex tori is an antiholomorphic homomorphism followed by a translation. As one varies over the parameter space, one obtains an "analytic family of antiholomorphic homomorphisms", and an "analytic family of translation parts". These are classified by certain cohomological invariants of the data defining the fibre variety which, together with the involution induced on the parameter space, constitute a complete set of invariants. In Chapter II, we obtain a necessary and sufficient condition for the existence of an antiholomorphic involution of a fibre variety with prescribed invariants (Theorem 1). In Chapter IV, we specialize our results to the case of analytic families of abelian varieties belonging to totally indefinite quaternion division algebras over totally real number fields. In this case, the

Received by the editors October 29, 1975 and, in revised form, October 3, 1977.

AMS (MOS) subject classifications (1970). Primary 14D10, 14K15, 14C30; Secondary 16A18, 30A58, $18 \mathrm{H} 10$.

Key words and phrases. Symmetric space, antiholomorphic, cohomology, cohomology with nonabelian coefficients, quaternion algebra, totally indefinite, algebraic number field, totally real, fibre variety, maximal order, ideal class group, connection, properly discontinuously. 
invariants have a natural description in terms of the division algebra, and the existence theorem specializes to an arithmetic criterion for the existence of involutions with prescribed invariants (Theorems 2 and 3).

In the course of determining some of these invariants, we have had to compute the group of holomorphic sections of these fibre varieties. This is done at the end of Chapter I and Chapter IV and yields an interesting class of algebraic cycles on the fibre-variety.

This paper was presented to the Mathematics Department of the State University of New York at Stony Brook in December of 1973 as the author's doctoral dissertation. The problem arose from a conversation between Michio Kuga and John Millson. Millson observed that the intrinsic theory of arithmetic groups acting on non-Hermitian symmetric spaces was much poorer than the theory for Hermitian symmetric spaces, but that it might be enriched by borrowing from the latter. He proposed that one investigate the equivariant embeddings of non-Hermitian symmetric spaces into Hermitian symmetric spaces and the restrictions to non-Hermitian symmetric spaces of holomorphic automorphic forms on the ambient Hermitian symmetric space. Professor Kuga reformulated the problem as the study of antiholomorphic involutions on Hermitian symmetric spaces and of the function fields of the corresponding real algebraic varieties. Approaching the problem rather optimistically, he anticipated that if one could develop a satisfactory arithmetic theory of automorphic functions for non-Hermitian symmetric spaces, its success would be entailed by the study of real forms of the objects which had guaranteed the success of the theory in the Hermitian case. These objects were, in the Hermitian case, the arithmetic quotients of bounded symmetric domains which could serve as moduli spaces of abelian varieties with additional structures. Ancillary to their study is the study of Hermitian symmetric spaces and the groups which act on them, while what is essential to the theory of canonical models of arithmetic quotients of bounded symmetric domains is the analysis of fibre systems of abelian varieties. And one is rewarded for one's trouble with the means of generating abelian extensions of totally imaginary quadratic extensions of totally real number fields. By introducing antiholomorphic involutions into theory, one might hope to have a similar theory of the abelian extensions of totally real number fields, using real forms of the canonical models. The study of these real forms would naturally involve the study of real forms of Hermitian symmetric spaces, and one might anticipate that the success of the theory would depend on studying the real forms of the fibre systems of abelian varieties.

Accordingly, Professor Kuga asked his students Harris Jaffee, Steve Kudla, and myself to study these problems for our theses. Harris Jaffee studied the antiholomorphic involutions of Hermitian symmetric spaces [5]. He obtained 
a complete list of the conjugacy classes of antiholomorphic involutions of the classical bounded symmetric domains as well as of their fixed point sets. Subsequently, he extended his results to the exceptional domains as well. Steve Kudla [4] studied the real forms of arithmetic quotients of a product of upper half planes belonging to a totally indefinite quaternion algebra over a totally real number field. In the case where the arithmetic group $\Gamma$ consists of all units of norm one of a maximal order of the division algebra, he obtained an explicit formula for the number of antiholomorphic involutions. This formula also gives the order of a nonabelian cohomology set $H^{1}(Z / 2 Z, \Gamma)$ and can be used to give explicit formulas for the results of Chapter IV of this paper. My task was to study the real forms of fibre systems of abelian varieties of Kuga-Satake type; the results of my investigations form the content of this paper. Hence, this paper may be said to derive from a suggestion of John Millson as well as from the rich legacy of the work of Shimura on complex multiplication and the arithmetic theory of automorphic forms. While we are happy to acknowledge the inspiring role which Shimura's contribution has played in our work, we are nevertheless obliged to report our disappointment that our acknowledgment is not a greater tribute to Professor Shimura. For although each of Professor Kuga's students solved the problem which was posed to him, the solution of these problems has not played any role in the construction of class fields over totally real fields. Were it otherwise, our results would be a worthy tribute to Professor Shimura.

After we had finished our dissertations, we learned that Professor Shimura [7] and his student Professor Shih [8] had been led by entirely different considerations to examine the problems of antiholomorphic involutions of abelian varieties and of bounded symmetric domains. They were concerned with the existence of rational points on abelian varieties and on arithmetic varieties, and observed that if such a variety admitted an antiholomorphic involution which had no fixed points, the corresponding real algebraic variety would have no real points and a fortiori no rational points.

\section{ChaPTER I. KUGA's Fibre VARIETIES}

1. Let $W$ be a vector-space of dimension $2 n$ over the field $\mathbf{R}$ of real numbers, let $W_{\mathrm{C}}=W \otimes_{R} \mathbf{C}$ denote its complexification, and let $\operatorname{Gr}\left(W_{\mathbf{C}}\right)$ denote the Grassmann manifold of complex $n$-planes $P$ in $\boldsymbol{W}_{\mathbf{C}}$.

If $X$ is any complex manifold and if $\varphi$ is a holomorphic mapping of $X$ into $\operatorname{Gr}\left(W_{\mathrm{C}}\right)$, then $\varphi$ determines a holomorphic vector-bundle $\beta(\varphi)$ over $X$ in the following way. The total space $E_{\varphi}$ of $\beta(\varphi)$ consists of all pairs $(x, v)$ belonging to $X \times W_{\mathrm{C}}$ for which the vector $v$ belongs to the $n$-plane $\varphi(x)$, and the projection mapping $E_{\varphi} \rightarrow^{\pi_{\varphi}} X$ is given by $\pi_{\varphi}(x, v)=x$. The addition and scalar multiplication are defined by the rules $\left(x, v_{1}\right)+\left(x, v_{2}\right)=\left(x, v_{1}+v_{2}\right)$, $c \cdot(x, v)=(x, c v)$. 
2. By a complex structure on the real vector space $W$, we mean a linear endomorphism of $W$ such that $J^{2}=-1_{W}$. If $J$ is given, we can define on $W$ the structure of a complex vector space by the rule $(a+b i) \cdot v=a \cdot v+$ $b J(v)$, for $a, b \in R$ and $v \in W$. We will denote by $(W, J)$ the complex vector-space so obtained.

If $J$ is a complex structure on $W$, then its $\mathbf{C}$-linear extension to $W_{\mathbf{C}}$ satisfies $J^{2}=1_{W_{c}}$, and we can write $W_{\mathrm{C}}=W_{J}^{+} \oplus W_{J}^{-}$, where $W_{J}^{+}$is the $i$-eigenspace of $J$ and $W_{J}^{-}$is the - $i$-eigenspace of $J . W_{J}^{+}$is a complex $n$-plane in $W_{\mathrm{C}}$ canonically associated to the complex structure $J$, and we say $W_{J}^{+}$ belongs to $J$.

It is easy to see that the mapping $w \rightarrow w-i J(w)$ defines an isomorphism of $x_{J}(W, J)$ onto $W_{J}^{+}$.

3. Suppose we are given a nondegenerate skew-symmetric bilinear form $B$ : $W \times W \mapsto \mathbf{R}$. Denote by $\mathcal{H}_{B}$ the set of all complex structures $J$ on $W$ for which the bilinear form $S_{J}: W \times W \rightarrow \mathbf{R}$ defined by $S_{J}(x, y)=B(x, J y)$ is symmetric and positive definite.

We have a canonical injection $\kappa: \mathcal{H}_{B} \rightarrow \operatorname{Gr}\left(W_{\mathrm{C}}\right)$ given by $J \mapsto \kappa(J)=W_{J}^{+}$ which maps $\mathcal{H}_{B}$ onto an open subset of the manifold of $n$-planes totally isotropic for $B$. Thus, $\mathcal{H}_{B}$ has a natural structure of complex manifold for which the mapping $\kappa$ is holomorphic.

4. Let $X$ be a bounded symmetric domain and let $\Gamma$ be a group acting holomorphically and properly discontinuously on $X$. Suppose that $\Gamma \backslash X$ is compact.

Let $\varphi_{0}: X \rightarrow \mathcal{H}_{B}$ be a holomorphic mapping, and let $\varphi=\kappa \circ \varphi_{0}: X \rightarrow$ $\operatorname{Gr}\left(W_{\mathbf{C}}\right)$. By the results of $\S 1, \varphi$ determines a holomorphic vector-bundle $\beta(\varphi)=\left(E_{\varphi}, \pi_{\varphi}\right)$ over $X$. The fibre $\pi_{\varphi}^{-1}(x)$ is just $\{x\} \times \varphi(x)$.

Using the remark at the end of $\S 2$, we define, for every $x \in X$, a complex linear isomorphism $\lambda_{x}:\left(W, \varphi_{0}(x)\right) \rightarrow \varphi(x)$ by the rule $\lambda_{x}(v)=v-i \varphi_{0}(x)(v)$.

Let $L$ be a lattice in $W$ such that $B$ is integer valued on $L \times L$. Let $\operatorname{Aut}(W, B, L)$ denote the group of all linear automorphisms $g$ of $W$ such that

(1) $g(L)=L$.

(2) $B(g(x), g(y))=B(x, y)$ for all $x, y \in W$.

Let $\rho: \Gamma \rightarrow \operatorname{Aut}(W, B, L)$ be a representation. Let $\tilde{\Gamma}$ denote the semidirect product of $\Gamma$ and $L$ via $\rho$. Explicitly, $\tilde{\Gamma}=\Gamma \times L$ with the group law defined by $\left(\gamma_{1}, l_{1}\right) \cdot\left(\gamma_{2}, l_{2}\right)=\left(\gamma_{1}, \gamma_{2}, l_{1}+\rho\left(\gamma_{1}\right)\left(l_{2}\right)\right)$.

We require that for $x \in X$ and $\gamma \in \Gamma$, we have $\varphi_{0}(\gamma \cdot x)=$ $\rho(\gamma) \circ \varphi_{0}(x) \circ \rho(\gamma)^{-1}$. It follows that $\varphi(\gamma \cdot x)=\rho(\gamma)(\varphi(x))$.

We define an action of $\tilde{\Gamma}$ on $E_{\varphi}$ by the rule $(\gamma, l) \cdot(x, v)=\left(\gamma \cdot x, \lambda_{\gamma \cdot x}(l)+\right.$ $\rho(\gamma)(v))$. It is straightforward to verify that in this way $\tilde{\Gamma}$ acts holomorphically and properly discontinuously on $E_{\varphi}$. 
Denote by $V$ the complex manifold $\tilde{\Gamma} \backslash E_{\varphi}$ and $U$ the complex manifold $\Gamma \backslash X$.

For $\tilde{\gamma}=(\gamma, l) \in \tilde{\Gamma}$, the diagram

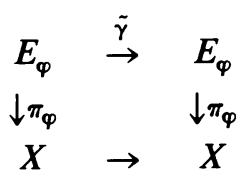

is commutative. It follows that $\pi_{\varphi}$ induces a holomorphic mapping $V \rightarrow^{\pi} U$.

Denote by $V \amalg V$ the set of all ordered pairs $\left(v_{1}, v_{2}\right)$ such that $\pi\left(v_{1}\right)=$ $\pi\left(v_{2}\right)$. For $\left(v_{1}, v_{2}\right) \in V \amalg V$, we can unambiguously define $\pi\left(v_{1}, v_{2}\right)$ to be $\pi\left(v_{1}\right)=\pi\left(v_{2}\right)$.

The addition in $E_{\varphi}$ determines a mapping $+: v \amalg v \rightarrow v$ which is holomorphic, and the 0 -section of $E_{\varphi}$ determines a holomorphic section $\eta$ : $U \rightarrow V$. Finally, the map $(x, v) \mapsto(x,-v)$ of $E_{\varphi}$ determines a holomorphic $\operatorname{map} \theta: V \rightarrow V$.

In this way, the sextuple $(V, \pi, U,+, \eta, \theta)$ can be viewed as an antyc family of compact complex Lie groups. Moreover, for each $x \in U, \pi^{-1}(x)$ has the structure of a polarized abelian variety.

Henceforth we will denote by $F_{x}$ the fibre $\pi^{-1}(x)$.

5. We recall now, for future reference, the following fact which is proved in [1]. $\tilde{\Gamma}$ acts on $X \times W$ by the rule $(\gamma, l)(x, u)=(\gamma \cdot x, l+\rho(\gamma) \cdot u)$.

LEMMA. There is a unique structure of holomorphic vector-bundle on $X \times W$ such that $\tilde{\Gamma}$ acts holomorphically and such that for all $x \in X$, the complex structure induced on the vector-space $\{x\} \times W$ is $\varphi_{0}(x)$.

Actually, we have essentially already proved the existence, for the mapping $\lambda: X \times W \rightarrow E_{\varphi_{0}}$ given by $(x, w) \rightarrow\left(x, \lambda_{x}(w)\right)$ is a bijection which induces on $X \times W$ the desired structure. For the uniqueness, see [1].

6. As we noted in the construction of $V$ in $\$ 4$, the group $\tilde{\Gamma}$ acts holomorphically and properly discontinuously on the space $E_{\varphi}$. Therefore, the subgroup $\Gamma$ of $\tilde{\Gamma}$ acts holomorphically and properly discontinuously on $E_{\varphi}$, and we denote by $V^{\#}$ the quotient space $\Gamma \backslash E_{\varphi}$. The projection mapping $\pi_{\varphi}$ : $E_{\varphi} \rightarrow X$ commutes with the action of $\Gamma$ on these spaces, and therefore induces a mapping $\pi^{\sharp}: V^{\sharp} \rightarrow U$. The pair $\left(V^{\sharp}, \pi^{\sharp}\right)$ is a holomorphic vector-bundle over $U$. It is proved in [1] that for all $q, H^{q}\left(U, V^{\sharp}\right)$ is canonically isomorphic to $H^{q}(\Gamma, W)$.

7. The mapping $\lambda: X \times W \rightarrow E_{\varphi}$ constructed in $\$ 5$ maps $X \times L$ onto a subspace $\tilde{\Lambda}$ of $E_{\varphi}$. If is evident that $\tilde{\Lambda}$ is a complex submanifold of $E_{\varphi}$ invariant under the action of $\Gamma$. Denote by $\Lambda$ the quotient space $\Gamma \backslash \tilde{\Lambda}$, which 
we view as a subspace of $V^{\sharp} . \Lambda$ is a sheaf over $U$. It is proved in [1] that for all $q, H^{q}(U, \Lambda)$ is canonically isomorphic to $H^{q}(\Gamma, L)$ and that the diagram

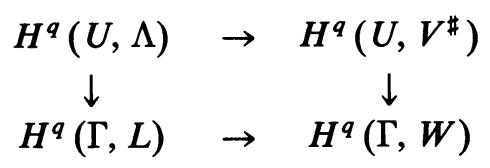

is commutative, where the horizontal arrows are coefficient homomorphisms and the vertical arrows are canonical isomorphisms.

In particular, we have $H^{q}\left(U, V^{\sharp}\right) \cong H^{q}(U, \Lambda) \otimes \mathbf{R}$.

8. In this section, we compute the group $\Xi$ of sections of the fibre variety $\pi$ : $V \rightarrow U$.

In the category of analytic families of complex Lie groups over $U$, the sequence

$$
0 \rightarrow \Lambda \rightarrow V^{\sharp} \rightarrow V \rightarrow 0 \text { is exact, }
$$

where $V^{\sharp} \rightarrow V$ is the natural mapping $\Gamma \backslash E_{\varphi} \rightarrow \tilde{\Gamma} \backslash E_{\varphi}$.

Denote by $\mathcal{V}^{\#}$ the sheaf of germs of holomorphic sections of $V^{\#}$, and by $\mathcal{V}$ the sheaf of germs of holomorphic sections of $V$.

As we noted in $\$ 7, \Lambda$ is already a sheaf. Then the sequence (*) determines an exact sequence

$$
0 \rightarrow \Lambda \rightarrow \mathcal{V}^{\sharp} \rightarrow \mathcal{T} \rightarrow 0
$$

of sheaves over $U$.

We therefore have an exact cohomology sequence

$$
0 \rightarrow H^{0}(U, \Lambda) \rightarrow H^{0}\left(U, \mathcal{V}^{\#}\right) \rightarrow H^{0}(U, \mathcal{V}) \stackrel{\delta}{\rightarrow} H^{1}(U, \Lambda) \rightarrow H^{1}\left(U, \mathcal{V}^{\#}\right)
$$

Of course, $H^{0}(U, \mathfrak{V})=\Xi$.

Using the isomorphisms described in $\S \S 6-7$, we have that

$$
0 \rightarrow H^{0}(\Gamma, L) \rightarrow H^{0}(\Gamma, W) \rightarrow \Xi \stackrel{\delta}{\rightarrow} H^{1}(\Gamma, L) \rightarrow H^{1}(\Gamma, W)
$$

is exact.

The kernel of $H^{1}(\Gamma, L) \rightarrow H^{1}(\Gamma, W)$ is just the torsion subgroup $H^{1}(\Gamma, L)^{\text {tors }}$ of $H^{1}(\Gamma, L)$ so that we obtain the following exact sequence

$$
0 \rightarrow \frac{H^{0}(\Gamma, W)}{H^{0}(\Gamma, L)} \rightarrow \Xi \rightarrow H^{1}(\Gamma, L)^{\text {tors }} \rightarrow 0 .
$$

This sequence splits since $H^{0}(\Gamma, W) / H^{0}(\Gamma, L)$ is divisible

$$
\therefore \Xi \cong \frac{H^{0}(\Gamma, W)}{H^{0}(\Gamma, L)} \times H^{1}(\Gamma, L)^{\text {tors }}
$$

In all the cases we will consider, $H^{0}(\Gamma, W)=0$, so that $\Xi \cong H^{1}(\Gamma, L)^{\text {tors }}$. 
Moreover, since the group $\Gamma$ is finitely generated, so is the group $H^{1}(\Gamma, L)^{\text {tors }}$. Therefore, for suitable $N, H^{1}(\Gamma, L)^{\text {tors }}$ consists entirely of $N$-torsion.

From the exact sequence of $\Gamma$-modules $0 \rightarrow L \rightarrow^{N} L \rightarrow L / N L \rightarrow 0$, we have the coefficient sequence

$$
H^{0}(\Gamma, L) \rightarrow H^{0}(\Gamma, L / N L) \stackrel{\delta}{\rightarrow} H^{1}(\Gamma, L) \stackrel{N}{\rightarrow} H^{1}(\Gamma, L) .
$$

Since we have $H^{0}(\Gamma, L)=0$, and the image of $\delta$ is the $N$-torsion in $H^{1}(\Gamma, L)$, we conclude that

$$
\Xi \cong H^{1}(\Gamma, L)^{\text {tors }} \cong H^{0}(\Gamma, L / N L) .
$$

9. The elements of $\Xi$ determine algebraic cycles in $V$. It would be interesting to study the homological properties of these cycles. For example, when do two elements of $\Xi$ determine homologous cycles?

We can describe these cycles explictly in a way which sheds more light on the isomorphism $\Xi \cong H^{0}(\Gamma, L / N L)$. Let $c \in L$ be an element which represents an element of $H^{0}(\Gamma, L / N L)$, so that $\rho(\gamma) \cdot(c) \equiv c(\bmod N L)$ for every $\gamma \in \Gamma$. Then $\rho(\gamma)(c / N) \equiv c / N(\bmod L)$ for every $\gamma \in \Gamma$.

It follows that there is a uniquely determined section $\omega$ of $V$ such that the diagram

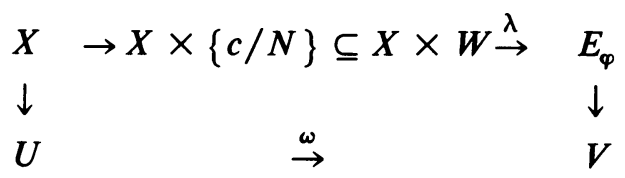

is commutative. Then $\omega$ and $c$ correspond to each other under the isomorphism (***).

10. We say that a holomorphic mapping $f: V \rightarrow V$ is an endomorphism of $V$ if $\pi \circ f=\pi$ and if for every $x \in U, f$ induces on the fibre $F_{x}$ an endomorphism of that complex Lie group. If $f$ is bijective, we say it is an automorphism of $V$. The set of all endomorphisms of $V$ forms a ring $\operatorname{End}(V)$, which we will always view as operating on $V$ from the right. The group of units of $\operatorname{End}(V)$ consists of the automorphisms of $C$, and is denoted by $\operatorname{Aut}(V)$.

\section{Chapter II. Existence of COMPLeX CONJUgations}

1. Let $M$ and $N$ be complex manifolds, and let $f: M \rightarrow N$ be a differentiable function. We say $f$ is antiholomorphic if $d f: T(M) \rightarrow T(N)$ induces conjugate-linear maps on the tangent spaces to $M$.

The following proposition is well known, but for the sake of completeness, we will give a proof. 
Proposition 1. Let $M$ and $N$ be complex tori and let $f: M \rightarrow N$ be an antiholomorphic mapping. Then there is a unique element $b \in N$ and $a$ unique antiholomorphic homomorphism $C: M \rightarrow N$ such that for all $x \in M$ we have $f(x)=C(x)+b$.

Proof. We may as well write $M=\tilde{M} / L_{1}, N=\tilde{N} / L_{2}$, where $\tilde{M}, \tilde{N}$ are complex vector spaces and $L_{1}$ and $L_{2}$ are lattices in $\tilde{M}$ and $\tilde{N}$ respectively. We can identify $\tilde{M}$ and $\tilde{N}$ with the universal covering spaces of $M$ and $N$ respectively. We can therefore cover $f$ with an antiholomorphic mapping $\tilde{f}$ : $\tilde{M} \rightarrow \tilde{N}$. For each $\lambda \in L$, and each $z \in \tilde{M}, \tilde{f}(z+\lambda)$ and $\tilde{f}(z)$ represent the same point of $N$. Therefore, there is a point $\varphi(\lambda, z) \in L$ such that $\tilde{f}(z+\lambda)=$ $\tilde{f}(z)+\varphi(\lambda, z)$. Since $\varphi$ is continuous with respect to $z$ and $L_{2}$ is discrete, $\varphi$ must actually be independent of $z$, so we can write $\varphi(\lambda, z)=\varphi(\lambda)$.

Then for all $\lambda \in L_{1}$ and $z \in \tilde{M}$, we have $d \tilde{f}(z+\lambda)=d \tilde{f}(z)$, so that $d \tilde{f}$ : $\tilde{M} \rightarrow \operatorname{Hom}(\tilde{M}, \tilde{N})$ is a periodic antiholomorphic function with period $L_{1}$. Therefore $d \tilde{f}$ is constant. This proves that $\tilde{f}$ is of the form $\tilde{C}+\tilde{b}$, where $\tilde{b} \in \tilde{N}$ and where $\tilde{C}: \tilde{M} \rightarrow \tilde{N}$ is a conjugate-linear mapping. The proposition follows at once. Q.E.D.

By a complex conjugation of a complex manifold $M$, we mean an antiholomorphic involution of $\boldsymbol{M}$, i.e., an antiholomorphic mapping $f: \boldsymbol{M} \rightarrow \boldsymbol{M}$ such that $f \circ f=1_{M}$.

2. Let $V \rightarrow^{\pi} U$ be a Kuga fibre variety, that is to say, a fibre system of abelian varieties over $U$ of the type discussed in $\$ 4$ of Chapter I. Our purpose is to study the complex conjugations of $V$.

Let us begin by remarking that a complex conjugation $\sigma$ of $V$ must preserve the fibres of $\pi$. To see this, let $x \in U$. Let $f: F_{x} \rightarrow U$ be the restriction of $\pi \circ \sigma$ to $F_{x} . f$ is antiholomorphic and is induced by an antiholomorphic mapping $\tilde{f}$ of the universal covering space of $F_{x}$, which is a complex vector space, into the universal covering space of $U$, which is $X$. Let $g: X \rightarrow X$ be an antiholomorphic isometry. Then $g \circ \tilde{f}$ is a bounded holomorphic function on a complex vector-space and is therefore constant. It follows that $f$ must be a constant map, say, $f(u)=y \in U$ for all $u \in F_{x}$. Then $f$ maps $F_{x}$ into $F_{y}$, which proves our assertion.

Let $\sigma$ be a complex conjugation of $V$. Since these fibres of $\pi$ are in 1-1 correspondence with the points of $U, \sigma$ induces a function $\sigma_{0}: U \rightarrow U$ such that the diagram

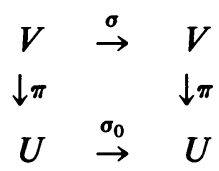


is commutative. Thus, $\sigma\left(F_{x}\right)=F_{\sigma_{0}(x)}$ for all $x \in U$. We can write $\sigma_{0} \circ \pi \circ \sigma_{0} \circ \eta$, so that $\sigma_{0}$ is antiholomorphic.

For every $x \in U, F_{x}$ is a complex torus, and $\sigma$ induces on $F_{x}$ an antiholomorphic mapping of $F_{x}$ into $F_{\sigma_{0}(x)}$. Applying the proposition of the preceding section, we conclude that for each $x \in U$, there is a unique element $b_{x}$ of $F_{\sigma_{0}(x)}$ and a unique antiholomorphic homomorphism $C_{x}: F_{x} \rightarrow F_{\sigma_{0}(x)}$ such that for all $u \in F_{x}$, we have $\sigma(u)=C_{x}(u)+b_{x}$.

We can therefore define a function $b: U \rightarrow V$ and a function $C: V \rightarrow V$ by the rules

$$
b(x)=b_{x}=\sigma \circ \eta(x)
$$

and $C(u)=C_{x}(u)=+\circ(\sigma(u), \theta \circ b \circ \pi(u))$, if $\pi(u)=x$.

It is evident from the descriptions that $b$ and $C$ are antiholomorphic, and we have $\sigma=C+(b \circ \pi)$.

For $u \in V$, if we put $x=\pi(u)$, we have

$$
u=\sigma^{2}(u)=C^{2}(u)+C\left(b_{x}\right)+b_{\sigma_{0}(x)} .
$$

In particular, if $u=\eta(x)$, we get

$$
\eta(x)=C\left(b_{x}\right)+b_{\sigma_{0}(x)}
$$

which we can rewrite as

$$
C \circ b=-b \circ \sigma_{0} .
$$

For arbitrary $u$, therefore, we have

$$
u=C^{2}(u)+\eta(x)=C^{2}(u),
$$

so that $C$ is also fibre preserving complex conjugation of $V$.

We call $C$ the homomorphism part of $\sigma$ and $b$ the translation part of $\sigma$.

In order to classify complex conjugations of $V$, it is therefore sufficient to classify all possible $C$ 's (i.e. all those which leave the image of $\eta$ invariant), and then to find all $b$ 's which, for a given $C$, satisfy (1).

3. The first invariant of the complex conjugation $\sigma$ is the complex conjugation $\sigma_{0}$ of $U$. As we noted above, the homomorphism part $C$ of $\sigma$ is also a complex conjugation of $V$ and it induces the same complex conjugation on $U$. It is natural to try to determine those complex conjugations of $U$ which are obtained in this way.

Let $\sigma_{0}$ be any complex conjugation of $U$, and let $\tilde{\sigma}_{0}$ be a lifting of $\sigma_{0}$ to an antiholomorphic mapping from $X$ to itself. Then $\tilde{\sigma}_{0}^{2}$ is a holomorphic covering transformation of $X$ over $U$, and therefore can be identified with an element $\gamma_{0}$ of $\Gamma$. Moreover, the mapping $\gamma \mapsto \tilde{\sigma}_{0} \gamma \tilde{\sigma}_{0}^{-1}$ defines an automorphism of $\Gamma$. 
THEOREM 1. A necessary and sufficient condition for the complex conjugation $\sigma_{0}$ of $U$ to be induced on $U$ by a complex conjugation of $V$ is that there exist a linear transformation $A: W \rightarrow W$ with the following four properties:

(1) $A^{2}=\rho\left(\gamma_{0}\right)$.

(2) A normalizes $\rho(\Gamma)$ and induces on $\Gamma$ the automorphism $\gamma \rightarrow \tilde{\sigma}_{0} \gamma \tilde{\sigma}_{0}^{-1}$.

(3) $A(L)=L$.

(4) For every $x \in X$, we have $A \circ \varphi_{0}(x)=-\varphi\left(\tilde{\sigma}_{0}(x)\right) \circ A$.

Proof. By the results of $\$ 2$ of this chapter, we may as well require that $\sigma_{0}$ be induced by a complex conjugation preserving the image of $\eta$.

As we noted in $\$ 5$ of Chapter $\mathrm{I}$, there is a unique structure of holomorphic vector-bundle on $X \times W \rightarrow X$ such that $\tilde{\Gamma}$ acts holomorphically on $X \times W$ and such that the complex structure induced on the vector-space $W$ is $\varphi_{0}(x)$.

The mapping $\lambda$ defined in that section determines a holomorphic isomorphism of that vector-bundle onto $E_{\varphi}$ which is compatible with the action of $\tilde{\Gamma}$. Therefore, we may view $X \times W$, with this $\tilde{\Gamma}$-action and complex structure, as the universal covering manifold of $V$.

Now suppose that $C$ is a complex conjugation of $V$ preserving $\eta$ and inducing $\sigma_{0}$ on $U$. Then there is a unique lifting of $C$ to an antiholomorphic mapping $\tilde{C}: X \times W \rightarrow X \times W$ such that the diagram

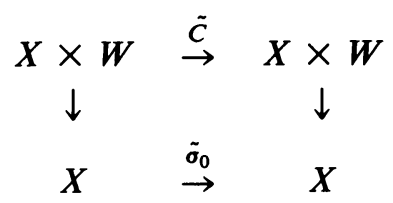

is commutative and such that for every $x \in X, \tilde{C}$ induces a real-linear transformation of the vector-space $\{x\} \times W$ onto $\left\{\tilde{\sigma}_{0}(x)\right\} \times W$. Therefore, we can write $\tilde{C}$ in the form $\tilde{C}(x, u)=\left(\tilde{\sigma}_{0}(x), A_{x} u\right)$, where $A_{x}: W \rightarrow W$ is $R$-linear. If $\tilde{\gamma} \in \tilde{\Gamma}$, then $\tilde{C} \tilde{\gamma} \tilde{C}$ is a holomorphic covering transformation of $X \times W$ over $V$, and therefore belongs to $\tilde{\Gamma}$. In particular, $\tilde{C}^{2}$ belongs to $\tilde{\Gamma}$, and for every $\tilde{\gamma} \in \tilde{\Gamma}, \tilde{C} \tilde{\gamma} \tilde{C}^{-1}=(\tilde{C} \tilde{\gamma} \tilde{C}) \tilde{C}^{-2}$ belongs to $\tilde{\Gamma}$. Actually, if we write $\tilde{C}^{2}=(\gamma, l)$, we must have $\gamma=\gamma_{0}$ and $l_{0}=0$, since $\tilde{C}$ covers $\tilde{\sigma}_{0}$ and preserves the 0 -section.

Therefore, $\tilde{C}^{2}=\left(\gamma_{0}, 0\right)$ and $\tilde{C}$ normalizes $\tilde{\Gamma}$. Since $\tilde{C}$ normalizes $\tilde{\Gamma}$, it follows that for any $l_{0} \in L$, we can find $(\gamma, l) \in \tilde{\Gamma}$ such that $\tilde{C}\left(0, l_{0}\right)=$ $(\gamma, l) \tilde{C}$.

For any $x \in X$, we have

$$
\begin{gathered}
\tilde{C}\left(0, l_{0}\right)(x, 0)=\tilde{C}(x, l)=\left(\tilde{\sigma}_{0} x, A_{x}\left(l_{0}\right)\right), \\
(\gamma, l) \tilde{C}(x, 0)=(\gamma, l)\left(\tilde{\sigma}_{0} x, 0\right)=\left(\gamma \tilde{\sigma}_{0}, l\right),
\end{gathered}
$$

so that $\left(\tilde{\sigma}_{0} x, A_{x}\left(l_{0}\right)\right)=\left(\gamma \tilde{\sigma}_{0}, l\right)$. 
Therefore $\gamma=1$ and $A_{x}\left(l_{0}\right)=l$, independently of $x$. Since $L$ contains a basis for $W$ over $R$, it follows that the transformation $A_{x}$ is independent of $x$. Therefore, we can write $A=A_{x}$. This is the linear transformation we are looking for.

What we have shown is that $A(L) \subseteq L$.

Since $\tilde{C}^{2}=\left(\gamma_{0}, 0\right)$, for every $(x, u) \in X \times W$ we have

$$
\begin{aligned}
\left(\gamma_{0} x, \rho\left(\gamma_{0}\right)(u)\right) & =\tilde{C}^{2}(x, u)=\tilde{C}\left(\tilde{\sigma}_{0} x, A(u)\right) \\
& =\left(\tilde{\sigma}_{0}^{2} x, A^{2}(u)\right)=\left(\gamma_{0} x, A^{2}(u)\right),
\end{aligned}
$$

so that $A^{2}=\rho\left(\gamma_{0}\right)$, which proves (1).

Since $A(L) \subseteq L$, we have $L=\rho\left(\gamma_{0}\right)(L)=A^{2}(L) \subseteq A(L) \subseteq L$, so that $A(L)=L$, which proves (3).

For $\gamma \in \Gamma$, we can find $\left(\gamma_{1}, l_{1}\right) \in \tilde{\Gamma} \ni \tilde{C}(\gamma, 0)=\left(\gamma_{1}, l_{1}\right) \tilde{C}$, since $\tilde{C}$ normalizes $\tilde{\Gamma}$. Then for $(x, u) \in X \times W$, we have $\tilde{C}(\gamma, 0)(x, u)=$ $\tilde{C}(\gamma x, \rho(\gamma) u)=\left(\tilde{\sigma}_{0} \gamma x, A \rho(\gamma)(u)\right)$ and $\left(\gamma_{1}, l_{1}\right) \tilde{C}(x, u)=\left(\gamma_{1}, l_{1}\right)\left(\tilde{\sigma}_{0} x, A(u)\right)=$ $\left(\gamma_{1} \tilde{\sigma}_{0} x, l_{1}+\rho\left(\gamma_{1}\right) A(u)\right)$. Therefore, we have $\left(\tilde{\sigma}_{0} \gamma x, A \circ \rho(\gamma)(u)\right)=\left(\gamma_{1} \tilde{\sigma}_{0} x, l_{1}\right.$ $\left.+\rho\left(\sim_{1}\right) \circ A(u)\right)$ for all $(x, u) \in X \times W$.

Thus we have

(a) $l_{1}=0$,

(b) $\gamma_{1}=\tilde{\sigma}_{0} \gamma \tilde{\sigma}_{0}^{-1}$

(c) $A \circ \rho(\gamma) \circ A^{-1}=\rho\left(\tilde{\sigma}_{0} \gamma \tilde{\sigma}_{0}^{-1}\right)$,

which proves (2).

Finally, since $\tilde{C}$ is antiholomorphic, the linear mapping $\left(W, \varphi_{0}(x)\right) \rightarrow$ $\left(W, \varphi_{0}\left(\tilde{\sigma}_{0}(x)\right)\right)$, given by $u \mapsto A_{u}$, must be antiholomorphic as well, i.e. it must be conjugate linear. Therefore, we must have $A \circ \varphi_{0}(x)=-\varphi_{0}\left(\tilde{\sigma}_{0}(x)\right) \circ A$ for all $x \in X$, which proves (4).

Conversely, suppose we are given a linear mapping $A: W \rightarrow W$ satisfying conditions (1)-(4). Define the mapping $\tilde{C}: X \times W \rightarrow X \times W$ by the rule $\tilde{C}(x, u)=\left(\tilde{\sigma}_{0} x, A(u)\right)$. Then $\tilde{C}^{-1}$ is given by $\tilde{C}^{-1}(x, u)=\left(\tilde{\sigma}_{0}^{-1} x, A^{-1}(u)\right)$.

By (1), $\tilde{C}^{2}=\left(\gamma_{0}, 0\right) \in \tilde{\Gamma}$. If $(\gamma, l) \in \tilde{\Gamma}$, then for all $(x, u) \in X \times W$ we have $\tilde{C}(\gamma, l) \tilde{C}^{-1}(x, u)=\left(\tilde{\sigma}_{0} \gamma \tilde{\sigma}_{0}^{-1} x, A(l)+A \circ \rho(\gamma) \circ A^{-1}(u)\right)$.

By (2), we have $A \circ \rho(\gamma) \circ A^{-1}=\rho\left(\tilde{\sigma}_{0} \gamma \tilde{\sigma}_{0}^{-1}\right)$, and by (3), $A(l) \in L$, so that the right-hand side of the above equation becomes

$$
\begin{aligned}
\left(\tilde{\sigma}_{0} \gamma \tilde{\sigma}_{0}^{-1} x, A(l)+\rho\left(\tilde{\sigma}_{0} \gamma \tilde{\sigma}_{0}^{-1}\right)(u)\right)=\left(\tilde{\sigma}_{0} \gamma \tilde{\sigma}_{0}^{-1}, A(l)\right)(x, u) & ; \\
& \therefore \tilde{C} \circ(\gamma, l) \circ \tilde{C}^{-1} \in \tilde{\Gamma},
\end{aligned}
$$

so $\tilde{C}$ normalizes $\tilde{\Gamma}$.

Since $\tilde{C}^{2} \in \tilde{\Gamma}$, we conclude that $\tilde{C}$ induces on $V$ a differentiable mapping $C: V \rightarrow V$ such that $C^{2}=1_{V}$. Obviously, $C$ preserves the fibres of $\pi$ and the image of $\eta$. Moreover, $C$ must induce the complex conjugation $\sigma_{0}$ on $U$. It only remains to prove that $C$ is antiholomorphic. 
Denote by $N$ the manifold $X \times W$ with the complex structure described in $\$ 5$ of Chapter I, and denote by $M$ the manifold $X \times W$ with the unique structure of complex manifold for which the mapping $\tilde{C}: M \rightarrow N$ is antiholomorphic. We only have to show that $M=N$, i.e. that these complex structures coincide. Let $g$ denote the almost complex structure on $X \times W$ determined by the complex manifold $N$, let $g^{\prime}$ denote the almost complex structure on $X \times W$ determined by the complex manifold $M$, and let $\mathscr{f}_{0}$ denote the almost complex structure on $X$.

It is easy to see that $g^{\prime}=-d \tilde{C}^{-1} \circ \mathcal{g} \circ d \tilde{C}$. In view of the uniqueness part of Lemma 1 of $\$ 5$, Chapter $\mathrm{I}$, in order to prove that $M=N$, or what is the same, that $g^{\prime}=\mathscr{g}$, we only have to verify that the almost complex structure $g^{\prime}$ has the other properties described in that lemma. This offers no difficulty, although the argument is somewhat tedious. The details are left to the reader.

4. Let $C$ be a complex conjugation of $V$ which preserves the fibres of $\pi$, the image of $\eta$, and which induces the complex conjugation $\sigma_{0}$ on $U$. The purpose of this section is to describe the set of translation parts $b$ which are compatible with $C$.

In order that $b: U \rightarrow V$ be a translation part, it is necessary and sufficient that $b$ be antiholomorphic, that $\pi \circ b=\sigma_{0}$, and that $C \circ b=-b \circ \sigma_{0}$. It is clear that the set of translation parts for $C$ forms a group under pointwise operations. Denote this group by $\operatorname{Trans}(C)$. If $b \in \operatorname{Trans}(C)$, then $b \circ \sigma_{0}$ is a holomorphic section of $V$, which we call $\omega_{b}$.

The mapping $b \mapsto \omega_{b}$ defines an isomorphism of $\operatorname{Trans}(C)$ onto the group of all sections $\omega \in \Xi$ such that $C \circ \omega \circ \sigma_{0}=-\omega$.

In Chapter I, $\S 8$, it was shown that $\Xi$ is isomorphic to $H^{0}(\Gamma, L / N L)$ for suitable $N$. Let $\tilde{\sigma}_{0}$ be a lifting of $\sigma_{0}$ to an antiholomorphic mapping of $X$ onto itself. By the results of $\S 3$, we can find a linear operator $A$ on $W$ which satisfies the conditions (1)-(4) of Theorem 1, and such that the mapping $\tilde{C}$ : $X \times W \rightarrow X \times W$, given by $\tilde{C}(x, u)=\left(\tilde{\sigma}_{0} x, A u\right)$, induces $C$ on $V$.

Let $\omega$ be a section of $V$, and let $\tilde{\omega}$ be a mapping $\tilde{\omega}: X \rightarrow X \times W$ which induces the section $\omega$ on $U$. By the results of $\S 9$, Chapter I, we can write $\tilde{\omega}$ in the form $\tilde{\omega}(x)=(x, c / N)$, where $c \in L$ satisfies the condition $\rho(\gamma)(c) \equiv c$ $(\bmod N L)$. Then the section $C \circ \omega \circ \sigma_{0}$ is induced on $U$ by the mapping $\tilde{C} \circ \tilde{\omega} \circ \tilde{\sigma}_{0}^{-1}$ (since $\sigma_{0}$ has order two), which is given by $\tilde{C} \circ \tilde{\omega} \circ \tilde{\sigma}_{0}^{-1}(x)=$ $(x, A(c) / N)$.

Therefore, the mapping $\omega \mapsto C \circ \omega \circ \sigma_{0}$ of $\Xi$ into itself has the following description in terms of $H^{0}(\Gamma, L / N L)$. By condition (3) of Theorem 1, $A(L)=L$, so $A(N L)=N L$. Therefore $A$ acts on $L / N L$. Moreover, since $A^{2} \in \rho(\Gamma), A^{2}$ must act trivially on $H^{0}(\Gamma, L / N L)$. Thus $A$ is an involution of $H^{0}(\Gamma, L / N L)$, and the group $\operatorname{Trans}(C)$ is isomorphic to the subgroup of 
$H^{0}(\Gamma, L / N L)$ consisting of elements $l \in H^{0}(\Gamma, L / N L)$ such that $A(l)=$ $-l$.

In Chapter IV, $\S 13$, we will determine the group $H^{1}(\Gamma, L)^{\text {tors }}$ for special choices of $\Gamma$ and $L$, so that the problem of determining $\operatorname{Trans}(C)$ is reduced to a reasonable computation.

If $C^{\prime}$ is a fibre preserving complex conjugation of $V$ which is conjugate to $C$ by an element $\alpha \in \operatorname{Aut}(V)$, say $C^{\prime}=\alpha C \alpha^{-1}$, then $\operatorname{Trans}\left(C^{\prime}\right)=$ $\alpha \operatorname{Trans}(C)$, so that the determination of $\operatorname{Trans}(C)$ depends only on the conjugacy class of $C$. In Chapter III, $\$ 8$, and Chapter IV, $\$ 10-11$, we will discuss how to classify $C$ up to conjugacy.

\section{Chapter III. COHOMOlogy WITH NONABELIAN COEFFICIENTS}

1. Let $G$ be a group. By a $G$-module, we mean a group $A$ together with a homomorphism of $G$ into the group of automorphisms of $A$. For $g \in G$, we denote the action of $g$ on an element $a \in A$ by $a \mapsto{ }^{8} a$.

By a 1-cocycle of $G$ valued in $A$, we mean a mapping $\alpha: G \rightarrow A$ such that for all $g, h \in G$ we have $\alpha(g h)=\alpha(g)^{g} \alpha(h)$. We denote by $Z^{1}(G, A)$ the set of all these 1-cocycles. This is a pointed set whose basepoint is the cocycle which maps $G$ onto the identity element of $A$. Given elements $\alpha, \alpha^{\prime}$ of $Z^{1}(G, A)$, we say that $\alpha$ and $\alpha^{\prime}$ are cohomologous if there exists an element $b \in A$ such that the equation $\alpha^{\prime}(g)=b^{-1} \alpha(g)^{8} b$ holds for every $g \in G$. This defines an equivalence relation on $Z^{1}(G, A)$. We denote by $H^{1}(G, A)$ the set of all the equivalence classes. This is a pointed set whose basepoint is the cohomology class of the basepoint of $Z^{1}(G, A)$. We call $H^{1}(G, A)$ the first cohomology set of $G$ with coefficients in $A$.

2. Let $A$ and $B$ be $G$-modules. By a morphism from $A$ to $B$, we mean a homomorphism $\phi: A \rightarrow B$ such that for all $g \in G$ and $a \in A$ we have $\phi\left({ }^{8} a\right)={ }^{8} \phi(a)$.

Let $\phi: A \rightarrow B$ be a morphism. If $\alpha: G \rightarrow A$ is a 1-cocycle, then $\phi \circ \alpha$ : $G \rightarrow B$ is easily seen to be a 1-cocycle valued in $B$. If $\alpha^{\prime}: G \rightarrow A$ is a 1-cocycle cohomologous to $\alpha$, then $\phi \circ \alpha^{\prime}$ and $\phi \circ \alpha$ are cohomologous as well.

In this way, $\phi$ determines a commutative diagram

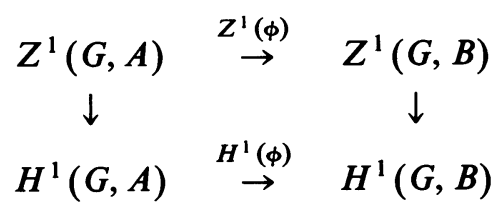

in the category of pointed sets.

In this way, $Z^{1}$ and $H^{1}$ are seen to be functors from the category of $G$-modules to the category of pointed sets. 
3. For the rest of this chapter, we will consider only the case where $G$ has two elements, say $G=\{1, g\}$. For any $G$-module $A$, we denote the action of $g$ on $A$ by $a \mapsto \bar{a}$ for all $a \in A$.

The mapping $\alpha \mapsto \alpha(g)$ defines a bijection between $Z^{1}(G, A)$ and the set of all elements $a \in A \ni \bar{a}=a^{-1}$. In view of this bijection, we will often refer to such elements of $A$ as cocycles. If $a, b \in A$ are such, they determine cohomologous cocycles if and only if there exists an element $c \in A$ such that $a=c^{-1} b \bar{c}$.

We will devote the rest of this chapter to examining some interesting examples of these cohomology sets.

4. Let $\tilde{M}$ be a differentiable manifold with a connection $\tilde{\nabla}$, and suppose that given any two points $x, y \in \tilde{M}$, there is a unique geodesic joining $x$ to $y$. Let $A$ be a group of connection preserving diffeomorphisms which act properly discontinuously on $\tilde{M}$. Denote by $M$ the quotient manifold and by $\nabla$ the connection induced on $M$ by $\nabla$. Let $\sigma$ be a diffeomorphism of $M$ onto itself, other than the identity, such that $\sigma^{2}=1_{M}$, and suppose that $\sigma$ preserves the connection $\nabla$.

Suppose further that $\sigma$ has a fixed point $x$, fixed throughout this paragraph and the next. Let $\tilde{x}$ be a point of $\tilde{M}$ representing $x$. We can find a lifting of $\sigma$

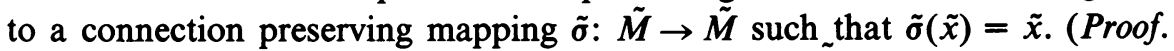
Let $\tilde{\sigma}^{\prime}$ be any lifting to $\tilde{M}$. It automatically preserves $\tilde{\nabla}$. Since $\tilde{x}$ represents a fixed point of $\sigma$, we can find $a \in A$ such that $\tilde{\sigma}^{\prime} \tilde{x}=a \tilde{x}$. Then we can take $\tilde{\sigma}=a^{-1} \tilde{\sigma}^{\prime}$.)

Then $\tilde{\sigma}^{2} \in A$, and fixes $\tilde{x}$, so $\tilde{\sigma}^{2}=1_{\tilde{M}}$. Moreover, $\tilde{\boldsymbol{\sigma}}$ normalizes $A$, so we can view $A$ as a $G$-module, $G=\left\{1_{\tilde{M}}, \tilde{\sigma}\right\}$. As in $\S 3$, we denote $\tilde{\sigma} a \tilde{\sigma}$ by $\bar{a}$ for all $a \in A$.

5. We will now define, for the situation described in $\$ 4$, a bijection between $H^{1}(G, A)$ and the set of path components of the set of fixed points of $\sigma$.

Let $x_{1}$ be a fixed point of $\sigma$, and let $\tilde{x}_{1}$ be a representative of $x_{1}$ in $\tilde{M}$. Let $\tilde{\sigma}_{1}$ be the unique lift of $\sigma$ to $\tilde{M}$ such that $\tilde{\sigma}_{1}\left(\tilde{x}_{1}\right)=\tilde{x}_{1}$. Then we can write $\tilde{\sigma}_{1}=a \tilde{\sigma}$ with $a \in A$. The element $a$ is uniquely determined and satisfies $a \bar{a}=a(\tilde{\sigma} a \tilde{\sigma})$ $=\tilde{\sigma}_{1}^{2}=1_{M}$. Thus, $a$ is a 1-cocycle, determined by $\tilde{x}_{1}$, of $G$ valued in $A$. If $\tilde{x}_{1}^{\prime}$ is another representative of $x_{1}$, we can write $\tilde{x}_{1}^{\prime}=b \tilde{x}_{1}$ with $b \in A$. If $\tilde{\sigma}_{1}^{\prime}$ is the lift of $\sigma$ to $\tilde{M}$ fixing $\tilde{x}_{1}^{\prime}$, then we have $b^{-1} \tilde{\sigma}_{1}^{\prime} b\left(\tilde{x}_{1}\right)=\tilde{x}_{1}=\tilde{\sigma}_{1}\left(\tilde{x}_{1}\right)$, which implies that $b^{-1} \tilde{\sigma}_{1}^{\prime}=\tilde{\sigma}_{1}$. If $a^{\prime}$ is the 1-cocycle determined by $\tilde{\sigma}_{1}^{\prime}$, then we have $\tilde{\sigma}_{1}^{\prime}=a^{\prime} \tilde{\sigma}$ so that $a \tilde{\sigma}=b^{-1} a^{\prime} \tilde{\sigma} b$, i.e. $a=b^{-1} a^{\prime} \tilde{\sigma} b \tilde{\sigma}=b^{-1} a \bar{b}$. Therefore the cohomology class we obtain from $x_{1}$ is independent of the representative $\tilde{x}_{1}$.

We claim that the cohomology class actually depends only on the path component of $x_{1}$ in the fixpoint set of $\sigma$. For suppose $x_{2}$ belongs to that component. Let $p$ be a path joining $x_{1}$ to $x_{2}$ such that $\sigma \circ p=p$. Let $\tilde{x}_{1}$ be a 
representative of $x_{1}$ in $\tilde{M}$, and let $\tilde{p}$ be the unique lift of $p$ to a path in $\tilde{M}$ beginning at $\tilde{x}_{1}$. Let $\tilde{x}_{2}=\tilde{p}(1)$. Let $\tilde{\sigma}_{1}, \tilde{\sigma}_{2}$ be the lifts of $\sigma$ which fix $\tilde{x}_{1}, \tilde{x}_{2}$ respectively, and write $\tilde{\sigma}_{1}=a \tilde{\sigma}$. Then since $\sigma$ fixes $p, \tilde{\sigma}_{1} \tilde{p}$ is a lift of $p$ to a path beginning at $\tilde{\sigma}_{1}\left(\tilde{x}_{1}\right)=\tilde{x}_{1}$, and so $\tilde{\sigma}_{1} \tilde{p}=\tilde{p}$. In particular, $\tilde{\sigma}_{1}\left(\tilde{x}_{2}\right)=$ $\tilde{\sigma}_{1}(\tilde{p}(1))=\tilde{p}(1)=\tilde{x}_{2}=\tilde{\sigma}_{2}\left(\tilde{x}_{2}\right)$. So $\tilde{\sigma}_{1}=\tilde{\sigma}_{2}$. Therefore $x_{1}$ and $x_{2}$ determine the same cohomology class.

This defines a mapping from the set of path components of the fixpoint set into $H^{1}(G, A)$. It remains to prove it is a bijection.

First we prove it is injective. Let $x_{1}, x_{2}$ be fixed points of $\sigma$ and suppose they determine the same cohomology class. Let $\tilde{x}_{1}, \tilde{x}_{2}$ be representatives of $x_{1}$, $x_{2}$ respectively, $\tilde{\sigma}_{1}, \tilde{\sigma}_{2}$ the corresponding lifts of $\sigma$, and $a$ and $b$ the corresponding cocycles. By hypothesis, these are cohomologous, so we can find $c \in A$ such that $a=c^{-1} b \bar{c}$.

Then $c \tilde{\sigma}_{1}=c a \tilde{\sigma}=b \bar{c} \tilde{\sigma}=b \tilde{\sigma} c=\tilde{\sigma}_{2} c$, so that $\tilde{\sigma}_{2}\left(c \tilde{x}_{1}\right)=c \tilde{x}_{1}$.

Let $\tilde{p}$ denote the unique geodesic joining $c \tilde{x}_{1}$ to $\tilde{x}_{2}$. Since $\tilde{\sigma}_{2}$ preserves $\tilde{\nabla}$, $\tilde{\sigma} \circ \tilde{p}$ is a geodesic and it joins $\tilde{\sigma}_{2}\left(c \tilde{x}_{1}\right)=c \tilde{x}_{1}$ to $\tilde{\sigma}_{2}\left(\tilde{x}_{2}\right)=\tilde{x}_{2}$. Therefore $\tilde{\sigma} \tilde{p}=\tilde{p}$, so that $\tilde{p}$ lies over a path $p$ in $M$ which is fixed by $\sigma$ and joins $x_{1}$ to $x_{2}$.

Finally, we prove the mapping is surjective. Let $a \in A$ be a 1-cocycle and let $\tilde{\sigma}_{1}=a \tilde{\sigma}$. We will be done if we can prove $\tilde{\sigma}_{1}$ has a fixed point. Let $\tilde{\boldsymbol{m}} \in \tilde{M}$. If $\tilde{m}$ is fixed by $\tilde{\sigma}_{1}$, we are done. If not, let $\tilde{p}$ be the unique geodesic joining $\tilde{m}$ to $\tilde{\sigma}_{1}(\tilde{m})$. Then $\tilde{\sigma}_{1} \circ \tilde{p}$ is the unique geodesic joining $\tilde{\sigma}_{1}(\tilde{m})$ to $\tilde{m}$, so we must have $\tilde{\sigma}_{1} \circ \tilde{p}(t)=\tilde{p}(1-t)$ for all $t \in[0,1]$. Then $\tilde{\sigma}_{1}\left(\tilde{p}\left(\frac{1}{2}\right)\right)=\tilde{p}\left(\frac{1}{2}\right)$ and we are done.

6. (a) If we take $\tilde{M}=X, A=\Gamma, \tilde{\nabla}$ to be the Riemannian connection for the natural metric on $X$, and take 0 to be a complex conjugation $\sigma_{0}$ of $U$, then we conclude that the components of the fixpoint set of $\sigma_{0}$ are classified by $H^{1}(G, \Gamma)$.

(b) If we take $\tilde{M}=W, A=L, \tilde{\nabla}$ to be the covariant constant connection on $W$, and $\sigma$ to be a complex conjugation on $M=W / L$ with respect to some complex structure on $M$, then the components of the fixpoint set of $\sigma$ are classified by $H^{1}(G, L)$. In this case, the components form a principal homogeneous space for the group $H^{1}(G, L)$.

(c) If we take $\tilde{M}=X \times W, A=\tilde{\Gamma}, \tilde{\nabla}=$ product of the two connections given in (a) and (b), and $\sigma$ to be a complex conjugation of $V$, then the components of the fixpoint set of $\sigma$ are classified by $H^{1}(G, \tilde{\Gamma})$.

7. Let $R$ be a ring with unity and let $a$ be a unit of $R$ contained in the center of $R$. Let $S$ denote the set of all elements $x \in R$ such that $x^{2}=a$, and suppose $S$ is nonempty. Let $x_{0}$ be a noncentral element of $S$ fixed throughout this discussion. Then $x_{0}$ is a unit, and the mapping $r \mapsto x_{0}^{-1} r x_{0}$ determines an automorphism $\psi$ of $R$ of order 2 which we denote by $r \mapsto \bar{r}$. If we take 
$G=\{1, \psi\}$, then the group $R^{x}$ of units of $R$ is a $G$-module.

On the other hand, $R^{x}$ acts by conjugation on $S$ since $a$ is in the center of $R$.

We will construct a bijection from the set $R^{x} \backslash S$ of $R^{x}$-conjugacy classes in $S$ onto $H^{1}\left(G, R^{x}\right)$. Let $x \in S$. We can write $x=x_{0} \alpha$ with $\alpha \in R^{x}$. Then $a=x^{2}=x_{0} \alpha x_{0} \alpha=a\left(x_{0}^{-1} \alpha x\right) \alpha=a \bar{\alpha} \alpha$, so that $\bar{\alpha} \alpha=1$ and $\alpha$ is a 1-cocycle valued in $R^{x}$. Conversly, if $\alpha$ is a 1-cocycle, then $x=x_{0} \alpha$ is clearly in $S$, so every cocycle is obtained in this way. Finally, two elements $x, y$ of $S$ are conjugate by an element $r$ of $R^{x}$ if and only if $r^{-1} x_{0}\left(x_{0}^{-1} x\right) r=x_{0}\left(x_{0}^{-1} y\right)$,

i.e. iff $\bar{r}\left(x_{0}^{-1} y\right) r^{-1}=x_{0}^{-1} x$,

i.e. iff the cocycles $x_{0}^{-1} x$ and $x_{0}^{-1} y$ are cohomologous.

8. Let $\sigma_{0}$ be a complex conjugation of $U$. Denote by $\Re\left(\sigma_{0}\right)$ the set of all complex conjugations of $V$ which preserve the image of $\eta$, and which induce on $U$ the complex conjugation $\sigma_{0}$. Suppose that $\Re\left(\sigma_{0}\right)$ is nonempty. Let $C_{0} \in \Re\left(\sigma_{0}\right)$ be an element fixed throughout this discussion. Let $G=\left\{1_{V}\right.$. $\left.C_{0}\right\}$. If $\alpha \in \operatorname{Aut}(V)$, then $C_{0} \alpha C_{0} \in \operatorname{Aut}(V)$ as well. In this way, $\operatorname{Aut}(V)$ is a $G$-module.

The group $\operatorname{Aut}(V)$ acts on the set $\mathscr{T}\left(\sigma_{0}\right)$ by conjugation. We will define a bijection of the set $\operatorname{Aut}(V) \backslash \Re\left(\sigma_{0}\right)$ of $\operatorname{Aut}(V)$ conjugacy classes in $\mathscr{T}\left(\sigma_{0}\right)$ onto $H^{\prime}(G, \operatorname{Aut}(V))$.

Given $C \in \Re\left(\sigma_{0}\right)$, we can write $C=\alpha C_{0}$ uniquely with $\alpha \in \operatorname{Aut}(V)$. Then $1_{V}=C^{2}=\alpha\left(C_{0} \alpha C_{0}\right)=\alpha \bar{\alpha}$, so $\alpha$ is a 1-cocycle. Conversely, it is clear that every 1-cocycle is obtained in this manner.

If $C, C^{\prime} \in \mathcal{T}\left(\sigma_{0}\right)$, and $C=\alpha C_{0}$ and $C^{\prime}=\beta C_{0}$, then $\alpha$ and $\beta$ are cohomologous iff $\exists \gamma \in \operatorname{Aut}(V)$ such that $\beta=\gamma^{-1} \alpha \bar{\gamma}$. This is equivalent to saying $C^{\prime}=\beta C_{0}=\gamma^{-1} \alpha \bar{\gamma} C_{0}=\gamma^{-1} \alpha C_{0} \gamma=\gamma^{-1} C \gamma$, i.e. that $C^{\prime}$ and $C$ are conjugate.

\section{Chapter IV. SOME SPECIAL CASES}

1. Let $D$ be a totally indefinite quaternion division algebra over a totally real number field $K$, and let $n=[K: \mathbf{Q}]$.

Let $\theta$ be an order in $D$, let $\theta^{x}$ be the group of units in $\theta$, let $\theta^{1}$ be the subgroup of $\theta^{x}$ consisting of units of reduced norm 1 , and let $\Gamma \subseteq \theta^{1}$ be a torsion-free subgroup of finite index in $\theta^{1}$.

We identify $D_{\mathbf{R}}=D \otimes_{\mathbf{Q}} \mathbf{R}$ with $M_{2}(\mathbf{R}) \times \cdots \times M_{2}(\mathbf{R})$, where the number of factors is $n$. Let $X$ equal the product $\mathcal{H} \times \cdots \times \mathcal{H}$ of $n$ copies of the upper half-plane.

Denote by $D_{\mathbf{R}}^{x}$ the group of units $\operatorname{GL}(2, \mathbf{R}) \times \cdots \times \mathrm{GL}(2, \mathbf{R})$ of $D_{\mathbf{R}}$, and by $D_{\mathbf{R}}^{1}$ the subgroup $\operatorname{SL}(2, \mathbf{R}) \times \cdots \times \operatorname{SL}(2, \mathbf{R})$ of $D_{\mathbf{R}}^{x}$.

We define an action of $D_{\mathbf{R}}^{x}$ on $X$ as follows. Given $g=\left(g_{1}, \ldots, g_{n}\right) \in D_{\mathbf{R}}^{x}$, where

$$
g_{\nu}=\left(\begin{array}{ll}
a_{\nu} & b_{\nu} \\
c_{\nu} & d_{\nu}
\end{array}\right)
$$


for $\nu=1, \ldots, n$, and $x=\left(x_{1}, \ldots, x_{n}\right)$, we put $g \cdot x=x^{\prime}=\left(x_{1}^{\prime}, \ldots, x_{n}^{\prime}\right)$ where

$$
x_{\nu}^{\prime}= \begin{cases}\frac{a_{\nu} x_{\nu}+b_{\nu}}{c_{\nu} x_{\nu}+d_{\nu}} & \text { if } a_{\nu} d_{\nu}-b_{\nu} c_{\nu}>0, \\ \frac{a_{\nu} \bar{x}_{\nu}+b_{\nu}}{c_{\nu} \bar{x}_{\nu}+d_{\nu}} & \text { if } a_{\nu} d_{\nu}-b_{\nu} c_{\nu}<0 .\end{cases}
$$

Then $D_{\mathrm{R}}^{x}$ acts as isometries of $X$ for the Bergmann metric.

Denote by $\Sigma_{n}$ the group of permutations on the set $\{1,2, \ldots, n\} . \Sigma_{n}$ acts as a group of automorphisms of $D_{\mathbf{R}}$ and as a group of isometries of $X$. In both cases, the action is given by the rule

$$
@ \cdot\left(x_{1}, \ldots, x_{n}\right)=\left(x_{@(1)}, \ldots, x_{@(n)}\right) \text { for @ } \in \Sigma_{n} .
$$

$\Sigma_{n}$ also acts as automorphisms of the product $\operatorname{PGL}(2, \mathbf{R}) \times \cdots \times$ $\operatorname{PGL}(2, \mathbf{R})$ of $n$ copies of $\operatorname{PGL}(2, \mathbf{R})$ in the same way. Denote by $G^{\#}$ the semidirect product $\Sigma_{n} \circ \operatorname{PGL}(2, \mathbf{R})^{n}$.

2. If $\psi$ is an automorphism of $D_{R}$, it induces an automorphism on the center $K_{\mathbf{R}}=K \otimes_{\mathbf{Q}} \mathbf{R}=\mathbf{R} \times \cdots \times \mathbf{R}$. Since $\mathbf{R}$ has only the identity automorphism, that automorphism of $K_{\mathbf{R}}$ must belong to $\Sigma_{n}$. Call that permutation@. Then@ ${ }^{-1} \circ \psi$ is an automorphism of $D_{\mathbf{R}}$ which is the identity on the center $K_{\mathrm{R}}$, and therefore induces an automorphism on each of the factors $M_{2}(\mathbf{R})$ of $D_{\mathrm{R}}$.

By the Skolem-Noether theorem, it follows that the automorphism $@^{-1} \circ \psi$ is an inner-automorphism $x \mapsto a x a^{-1}$ with $a \in D_{\mathbf{R}}^{x}$. It follows that the group $\operatorname{Aut}\left(D_{\mathbf{R}}\right)$ is the semidirect product of $\Sigma_{n}$ with the group of inner-automorphisms of $D_{\mathbf{R}}$. The group of inner-automorphisms is just $D_{\mathbf{R}}^{x}$ modulo its center, which is $\operatorname{GL}(2, \mathbf{R}) \times \cdots \times \mathrm{GL}(2, \mathbf{R}) / \mathbf{R}^{x} \times \cdots \times \mathbf{R}^{x}=$ $\operatorname{PGL}(2, \mathbf{R}) \times \cdots \times \operatorname{PGL}(2, \mathbf{R})$.

Thus $\operatorname{Aut}\left(D_{\mathbf{R}}\right)$ is isomorphic to $G^{\#}$.

Let $\psi$ be an automorphism of $D_{\mathbf{R}}$ and let $\omega$ be the corresponding isometry of $X . \psi$ induces an automorphism on $D_{\mathbf{R}}^{x}$, and therefore on the group $D_{\mathbf{R}}^{x}$ modulo its center, which is $\operatorname{PGL}(2, \mathbf{R}) \times \cdots \times \operatorname{PGL}(2, \mathbf{R})$. On the other hand, the inner automorphism of $G^{\#}$ determined by $\omega$ leaves $\operatorname{PGL}(2, \mathbf{R})$ $\times \cdots \times \operatorname{PGL}(2, \mathbf{R})$ invariant and induces an automorphism on that group which coincides with the automorphism determined by $\psi$. This is easily seen to be true. If $\psi \in \Sigma_{n}$, it is obvious. If $\psi \in \operatorname{PGL}(2, \mathbf{R}) \times \cdots \times \operatorname{PGL}(2, \mathbf{R}), \psi$ acts by conjugation on $D_{\mathrm{R}}$, and therefore also acts by conjugation on $G^{\sharp}$, so it obviously coincides with the conjugation by $\omega$. We will denote by $\Gamma^{\prime}$ the image of $\Gamma$ in $\operatorname{Isom}(X)$. Our hypotheses imply that $\Gamma$ is mapped isomorphically onto $\Gamma^{\prime}$. 
3. The elements of $D_{\mathbf{R}}^{x}$ act as isometries of $X$. The kernel of this action is again the center of $D_{\mathbf{R}}^{x}$, so that the action factors through $\operatorname{PGL}(2, \mathbf{R})$ $\times \cdots \times \operatorname{PGL}(2, \mathbf{R})$. The subgroup $\operatorname{PSL}(2, \mathbf{R}) \times \cdots \times \operatorname{PSL}(2, \mathbf{R})$ acts transitively on $X$. If $\sigma$ is an isometry of $X$, it can be written uniquely as the composition of an element $g \in \operatorname{PGL}(2, \mathbf{R}) \times \cdots \times \operatorname{PGL}(2, \mathbf{R})$ and $a$ permutation@ @ $\in \Sigma_{n}$. In this way, the group $\operatorname{Isom}(X)$ of isometries of $X$ is canonically isomorphic to $G^{\#}$.

It follows that $\operatorname{Aut}\left(D_{\mathbf{R}}\right)$ and $\operatorname{Isom}(X)$ are isomorphic.

4. Denote by $x \rightarrow \bar{x}$ the canonical involution of $D$ given by $\bar{x}=\operatorname{tr}(x)-x$, where $\operatorname{tr}: D \rightarrow K$ is the reduced trace. Let $S \in \theta$ be a nonzero element such that $\bar{S}=-S$. Define a skew-symmetric $\mathbf{Q}$-bilinear form $B_{0}: D \times D \rightarrow \mathbf{Q}$ by the rule $B_{0}(x, y)=\operatorname{tr}_{D / Q}(S \bar{x} y) . B_{0}$ is easily seen to be integer-valued on $\theta \times \theta$ and nondegenerate.

Put $W=D_{\mathbf{R}}, B=\mathbf{R}$-bilinear extension of $B_{0}$ to $D_{\mathbf{R}}$, and take $L=2$-sided $\theta$-ideal in $\theta$.

For the action of $D_{\mathbf{R}}^{x}$ on $X$ defined in $\S 1$ of this chapter, $\Gamma$ acts holomorphically and properly discontinuously on $X$. Denote by $\rho$ the leftregular representation of $D_{\mathbf{R}}$ on itself. We will also denote by $\rho$ the restrictions of the left-regular representation to $D_{\mathbf{R}}^{x}$ and to $\Gamma$.

For $\gamma \in \Gamma \subseteq \theta^{1}$, we have $\bar{\gamma} \gamma=1$. Therefore, for any $x, y \in D$ we have $B_{0}(\gamma x, \gamma y)=\operatorname{tr}_{D / Q}(S(\overline{\gamma x}) \gamma y)=\operatorname{tr}_{D / Q}(S \bar{x} \bar{\gamma} x y)=\operatorname{tr}_{D / Q}(S \bar{x} y)=B_{0}(x, y)$, so $\rho(\gamma)$ preserves $B_{0}$ and therefore $B$ as well.

Moreover, $\rho(\gamma) L=\gamma \mathfrak{A}=\mathfrak{A}=L$ and since $\mathfrak{A} \subseteq \mathcal{O}, B$ is integer-valued on $L \times L . \therefore \rho$ maps $\Gamma$ into $\operatorname{Aut}(W, B, L)$.

We define $\varphi_{0}: \quad X \rightarrow \mathcal{H}_{B}$ as follows. Denote by $J$ the element $\left(\left(\begin{array}{cc}0 & 1 \\ -1 & 0\end{array}\right), \ldots,\left(\begin{array}{cc}0 & 1 \\ -1 & 0\end{array}\right)\right) \in D_{\mathrm{R}}$, and denote by $z_{0}$ the point $(i, i, \ldots, i)$ of $X$. For $z \in X$, we can find $g \in D_{\mathbf{R}}^{1}$ such that $g \circ z_{0}=z$. We then define $\varphi_{0}(z)=$ $\rho\left(g J g^{-1}\right)=\rho\left(J^{t} g^{-1} g^{-1}\right)$. It is easy to see that this is well defined.

For $\gamma \in \Gamma$, we have $\varphi_{0}(\gamma z)=\rho\left(\gamma g J g^{-1} \gamma^{-1}\right)=\rho(\gamma) \varphi_{0}(z) \rho(\gamma)^{-1}$. It is easy to see that the mapping $\varphi_{0}$ is holomorphic. We will omit the details.

5. In this section, we prove that $\Gamma$ contains a basis for $D$ over $\mathbf{Q}$. Let $D_{0}=\mathrm{Q}[\Gamma]$ denote the Q-linear span of $\Gamma$. Then $D_{0}$ is a division algebra over Q. We would like to prove that $D=D_{0}$. Let $\rho_{0}$ denote the left-regular representation of $\Gamma$ on $D_{0}$ and on $D_{0 R}=D_{0} \otimes_{\mathbf{Q}}$ R. We can view $D$ as a left-vector-space of dimension $m$ over $D_{0}$, so that $D_{\mathrm{R}} \cong D_{0 \mathrm{R}} \times \cdots \times D_{0 \mathrm{R}}$ ( $m$ copies). Therefore $D_{\mathrm{R}}^{x} \cong D_{0 \mathrm{R}}^{x} \times \cdots \times D_{0 \mathrm{R}}^{x}$, and if we denote by $Z$ the center of $D_{0 \mathrm{R}}^{x}$, we have that $\operatorname{PSL}(2, \mathbf{R}) \times \cdots \times \operatorname{PSL}(2, \mathbf{R})(n$ copies) is isomorphic to the connected component of $\left(D_{0 \mathrm{R}}^{x} / Z\right) \times \cdots \times\left(D_{0 \mathrm{R}}^{x} / Z\right)$. Using a basis for $D$ over $D_{0}$, we can view $\rho$ as the direct sum of $m$ copies of $\rho_{0}$. Now, $\Gamma$ acts on $\operatorname{PSL}(2, \mathbf{R}) \times \cdots \times \operatorname{PSL}(2, \mathbf{R})$ by conjugation, and it is 
not difficult to see that this corresponds, under the above isomorphism, to the action of $\Gamma$ on $\left(D_{0 \mathrm{R}}^{x} / Z\right) \times \cdots \times\left(D_{0 \mathrm{R}}^{x} / Z\right)$ given by

$$
\gamma \cdot\left(\delta_{1} Z, \delta_{2} Z, \ldots, \delta_{m} Z\right)=\left(\rho_{0}(\gamma) \delta_{1} Z, \ldots, \rho_{0}(\gamma) \delta_{m} Z\right) .
$$

Let $\pi_{i}, i=1, \ldots, m$, denote the projection of $\left(D_{0 \mathrm{R}}^{x} / Z\right) \times \cdots \times\left(D_{0 \mathrm{R}}^{x} / Z\right)$ onto its $i$ th factor. Let $H=\operatorname{PSO}(2) \times \cdots \times \operatorname{PSO}(2) \subseteq \operatorname{PSL}(2, \mathbf{R}) \times \cdots \times$ $\operatorname{PSL}(2, \mathbf{R}) . H$ is a maximal compact subgroup, and its image in $\left(D_{0 R}^{x} / Z\right)$ $\times \cdots \times\left(D_{0 \mathrm{R}}^{x}\right)$ which we call $H^{\prime}$, must be a maximal compact subgroup. Therefore, $H^{\prime}=\pi_{1}\left(H^{\prime}\right) \times \cdots \times \pi_{n}\left(H^{\prime}\right)$.

Let $X_{i}=\left(D_{0 \mathrm{R}}^{x} / Z\right) / \pi_{i}\left(H^{\prime}\right)$. Then we have $X \cong X_{1} \times \cdots \times X_{m}$, and this isomorphism is compatible with the action of $\Gamma$.

Now suppose that $m$ is greater than 1 .

Then $\Gamma \backslash X$ can be viewed as a fibre bundle over $\Gamma \backslash X_{1}$ whose fibre is $X_{2} \times \cdots \times X_{m}$. Since $\Gamma \backslash X$ is compact, $\Gamma \backslash X_{1}$ and $X_{2} \times \cdots \times X_{m}$ must also be compact. However, since each $\pi_{i}\left(H^{\prime}\right)$ is compact, $X_{2} \times \cdots \times X_{m}$ is compact if and only if $D_{0 R}^{x} / Z$ is. But that would imply that $\operatorname{PSL}(2, \mathbf{R})$ $\times \cdots \times \operatorname{PSL}(2, \mathbf{R}) \cong\left(D_{0 \mathrm{R}}^{x} / Z\right) \times \cdots \times\left(D_{0 \mathrm{R}}^{x} / Z\right)$ is compact, which is a contradiction. Therefore $m=1, D_{0}=D$, and we are done.

6. Let $\sigma_{0}$ be an antiholomorphic involution of $U$. Let $\tilde{\sigma}_{0}$ be a lifting of $\sigma_{0}$ to an antiholomorphic mapping of $X$ into itself. It is not hard to prove that $\tilde{\sigma}_{0}$ must be an isometry of $X$ for the Bergmann metric. Moreover, we can choose $\tilde{\sigma}_{0}$ to be an involution of $X$ if and only if $\sigma_{0}$ has a fixed point. Let $\psi$ be the automorphism of $D_{\mathrm{R}}$ corresponding to $\tilde{\sigma}_{0}$ under the canonical isomorphism of §3. Then $\psi$ must leave $\Gamma$ invariant.

LeMMA. $\psi \circ \varphi_{0}(x)=-\varphi_{0}\left(\tilde{\sigma}_{0}(x)\right) \circ \psi$ for every $x \in X$.

Proof. By $\S 3$, we can write $\psi=@ \circ T_{h}$, where $h \in D_{\mathbf{R}}^{x}$, and $T_{h}$ denotes the inner-automorphism determined by $h$. Since $\tilde{\sigma}_{0}$ is antiholomorphic, we can choose $h=\left(h_{1}, \ldots, h_{n}\right) \in D_{\mathrm{R}}^{x}$ so that $\operatorname{det}\left(h_{i}\right)=-1$ for $i=1, \ldots, n$.

Let $\alpha=\left(\left(\begin{array}{ll}1 & 0 \\ 0 & -1\end{array}\right), \ldots,\left(\begin{array}{ll}1 & 0 \\ 0 & -1\end{array}\right)\right) \in D_{\mathbf{R}}$. Let $x \in X$. We can write $x=g \cdot z_{0}$, where $g=\left(g_{1}, \ldots, g_{n}\right) \in D_{\mathrm{R}}^{1}$, and $z_{0}=(i, i, \ldots, i)$ as in $\S 4$.

Then it is easy to check that $h \cdot z=(h g \alpha) \circ z_{0}$, since $\alpha \circ z_{0}=z_{0}$, and the element $h g \alpha$ belongs to $D_{\mathbf{R}}^{1}$. Therefore $\varphi_{0}(h \circ z)=\rho\left(h g \alpha J \alpha g^{-1} h^{-1}\right)=$ $-\rho\left(h g J g^{-1} h^{-1}\right)$. For $y \in D_{\mathbf{R}}$, we have then

$$
\begin{aligned}
\varphi_{0}(h \cdot z)(y) & =-h g J g^{-1} h^{-1} y \\
& =-h\left(\left(g J g^{-1}\right)\left(h^{-1} y h\right)\right) h^{-1}=-T_{h} \circ \varphi_{0}(z) \circ T_{h}^{-1}(y),
\end{aligned}
$$

so that $\varphi_{0}(h \cdot z) T_{h}=-T_{h} \circ \varphi_{0}(z)$. Finally 


$$
\begin{aligned}
\varphi_{0}\left(\tilde{\sigma}_{0} \circ z\right) & =\varphi_{0}\left(@ \circ\left(T_{h} g \alpha\right) \circ z_{0}\right) \\
& =\rho\left(@(h g \alpha) J @(h g \alpha)^{-1}\right) \\
& =@ \circ \rho\left((h g \alpha) J(h g \alpha)^{-1}\right) \circ @^{-1} \\
& =@ \circ \varphi_{0}(h \cdot z) \circ @^{-1}=-@ \circ T_{h} \circ \varphi_{0}(z) \circ h^{-1} \circ @^{-1}
\end{aligned}
$$

so $\varphi_{0}\left(\tilde{\sigma}_{0}^{\circ} \circ\right) \circ \psi=-\psi \circ \varphi_{0}(z)$, as desired.

7. Let $\sigma_{0}$ be as in $\S 6$. Let $V$ be the Kuga fibre variety defined in $\$ 4$ of this chapter, where we take $\mathfrak{A}=\mathcal{\theta}$. Our object in this section is to prove the following result.

THEOREM 2. Suppose that $\sigma_{0}$ has a fixpoint. Let $\tilde{\sigma}_{0}$ be a complex conjugation of $X$ covering $\sigma_{0}$, and let $\psi$ be the corresponding automorphism of $D_{\mathbf{R}}$. Then $\sigma_{0}$ is induced by a complex conjugation of $V$ if and only if $\psi(\theta)=\theta$.

Proof. $(\Rightarrow)$ Suppose $\sigma_{0}$ is so induced. Then by Theorem 1 of Chapter II, $\S 3$, and the results of $\$ 2$ of this chapter, we can find an operator $A$ on $W$ such that

(1) $A^{2}=1_{W}$.

(2) $A$ normalizes $\rho(\Gamma)$ and induces the automorphsim $\psi$ on $\Gamma$.

(3) $A(\theta)=\theta$.

(4) $A \circ \varphi_{0}(x)=-\varphi_{0}\left(\tilde{\sigma}_{0} x\right) \circ A$ for all $x \in X$.

Let $B=A \circ \psi$. Since $A^{2}=\psi^{2}=1_{W}$, for any $\gamma \in \Gamma$ and any $y \in W$, we have

$$
\begin{aligned}
B \circ \rho(\gamma) \circ B^{-1}(y) & =A \circ \psi \circ \rho(\gamma) \circ \psi \circ A(y) \\
& =A(\psi(\gamma) A(y))=A \circ \rho(\psi(\gamma)) \circ A(y) \\
& =\rho\left(\psi^{2} \gamma\right)(y)=\rho(\gamma)(y) .
\end{aligned}
$$

Thus, $B$ commutes with the elements of $\rho(\Gamma)$. By the results of $\S 6$ of this chapter, $\Gamma$ contains a basis for $D_{\mathbf{R}}$ over $\mathbf{R}$, so $B$ commutes with the elements of $\rho\left(D_{\mathrm{R}}\right)$. Therefore $B$ has the form $x \mapsto x b$ with $b \in D_{\mathrm{R}}$. Therefore, $A$ is given by $A(x)=\psi(x) b$ for all $x \in D_{\mathbf{R}}$. By (3), we have $\theta=A(\theta)=\psi(\theta) b=$ $\psi(\theta) \psi(\theta) b=\psi(\theta) \theta$, since $\psi(\theta)$ is an order, and therefore $\psi(\theta) \subseteq \theta$. Applying $\psi$, we get $\theta=\psi^{2}(\theta) \subseteq \psi(\theta)$, so $\psi(\theta)=\theta$.

$(\Leftarrow)$ Suppose $\psi(\theta)=\theta$. Let $A=\psi$. Then we claim (1)-(4) are satisfied. For (1) and (2), it is obvious, (3) holds by hypothesis, and (4) holds by the lemma of $\$ 6$.

8. In the situation described in $\$ \S 4-5$, we now take $\theta$ to be a maximal order. Let $T \in D^{x}$, and suppose that $T \Gamma T^{-1}=\Gamma$. Then the action of $T$ on $X$ induces a mapping on $U=\Gamma \backslash X$. Moreover, if we require that $T^{2} \in K^{x} \Gamma$ and that the reduced norm $\nu(T)$ of $T$ be a totally negative element of $K$, then that induced mapping will be a complex conjugation of $U$, which we call $\sigma_{0}$. 
THeOREM 3. In order that $\sigma_{0}$ be induced by a complex conjugation of $V$, it is necessary that $T \vartheta=\mathcal{O} T$ and that $K(\sqrt{-\nu(T)})$ be embeddable in $D$ over $K$.

If the ideal class group of $K$ is generated by the primes of $K$ at which $D$ is ramified, then these conditions are also sufficient.

Proof. Let $c$ be an element of $K \otimes_{\mathbf{Q}} \mathbf{R}$ whose square is $-\nu(T)$, and let $\tilde{\sigma}_{0}=c^{-1} T$. If we write $\tilde{\sigma}_{0}=\left(\tilde{\sigma}_{1}, \ldots, \tilde{\sigma}_{n}\right)$, then $\operatorname{det}\left(\tilde{\sigma}_{i}\right)=-1$, for $i=$ $1, \ldots, n$, and $\tilde{\sigma}_{0}$ determines an antiholomorphic mapping of $X$ into itself which covers $\sigma_{0}$. Suppose $\sigma_{0}$ is induced by a complex conjugation of $V_{0}$. Then by the results of Chapter II, $\S 3$, we can find an operator $A$ on $W$ such that:

(1) $A^{2}=\rho\left(\tilde{\sigma}_{0}\right)^{2}$.

(2) $A$ normalizes $\rho(\Gamma)$ and induces on $\Gamma$ the same automorphism that $\tilde{\sigma}_{0}$ does.

(3) $A(\mathfrak{I})=\mathfrak{A}$.

(4) $A \varphi_{0}(x)=-\varphi_{0}\left(\tilde{\sigma}_{0} \cdot z\right) A$.

Let $A_{0}=\rho\left(\sigma_{0}\right)$ and let $B=A_{0}^{-1} A$. Since $A$ and $A_{0}$ induce the same automorphism on $\rho(\Gamma)$, it follows that $B$ commutes with the elements of $\rho(\Gamma)$. Therefore, $B$ commutes with the elements of $\rho\left(D_{\mathbf{R}}\right)$ and is of the form $x \mapsto x b$, with $b \in D_{\mathbf{R}}$. Since $B$ commutes with the elements of $\rho\left(D_{\mathbf{R}}\right)$, in particular, $B$ commutes with $A_{0}$, so $A$ commutes with $A_{0}$. Therefore, since $A^{2}=A_{0}^{2}$, by (1) we have $B^{2}=1_{W}$, so $b^{2}=1$.

By (3), we have $\mathfrak{A}=A(\mathfrak{U})=A_{0} B(\mathfrak{U})=\tilde{\sigma}_{0} \mathfrak{U} b$. Let $\beta=c^{-1} b$. Then $\mathfrak{A}=$ $\tilde{\sigma}_{0} \mathfrak{A} b=T \mathfrak{A} \beta$, so that $\beta \in D^{x}, \beta^{-2}=c^{2} b^{-2}=-\nu(T)$, so $K(\sqrt{-\nu(T)})$ embeds in $D$.

Since $\theta$ is maximal, $\theta$ is the left order of $\mathscr{A}$, and $T \Theta T^{-1}$ is maximal. Then $\mathfrak{A}=T \mathfrak{A} \beta=T \mathcal{A} \beta=T \vartheta T^{-1} T \mathfrak{A} \beta=T \vartheta T^{-1} \mathfrak{A}$ so that $T \vartheta T^{-1} \subseteq \mathcal{\theta}$, which implies $T \theta=\theta T$.

Conversely, let $\mathscr{P}_{1}, \ldots, \mathscr{P}_{r}$ be the distinct primes of $K$ where $D$ is ramified, and suppose that they generate the ideal class group of $K$. We will prove that the conditions $T \Theta=\Theta T$ and $K(\sqrt{-\nu(T)})$ embeddable in $D$ imply that $\sigma_{0}$ is induced on $U$ by a complex conjugation $\sigma$ of $V$.

We can, by multiplying $T$ by an element of $K^{x}$ if necessary, assume without loss of generality that $T \in \theta$ and there is an element $\delta \in \theta$ such that $\delta^{2}=-\nu(T)=c^{2}$. Put $b=c^{-1} \delta, \beta=c^{-2} \delta$, and let $B \in \operatorname{GL}\left(D_{\mathbf{R}}\right)$ be defined by $B(x)=x b$ for all $x \in D_{\mathbf{R}}$. Then $B$ commutes with $\rho\left(D_{\mathbf{R}}\right)$. Let $A_{0}=\rho\left(\tilde{\sigma}_{0}\right)$ and let $A=A_{0} B$, so that for $x \in D_{R}$, we have $A(x)=\tilde{\sigma}_{0} x b$. We clearly have $B^{2}=1_{D_{R}}$.

We will prove that $A$ satisfies the condition (1)-(4) given in the direct part of this proof.

Proof of (1). Since $A_{0} \in \rho\left(D_{\mathrm{R}}\right), A_{0}$ and $B$ commute. Therefore, $A^{2}=$ $\left(A_{0} B\right)^{2}=A_{0}^{2} B^{2}=A_{0}^{2}=\rho\left(\tilde{\sigma}_{0}\right)^{2}$. 
Proof of (2). Since $B$ commutes with $\rho(\Gamma)$, this is obvious.

Proof of (3). For each prime ideal $B$ of $K$, there is a unique maximal ideal

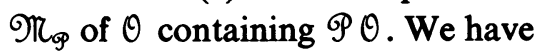

$$
\mathscr{P} \mathcal{O}=\left\{\begin{array}{l}
\Re_{\mathscr{P}} \text { if } D \text { is unramified at } \mathscr{P}, \\
\mathfrak{K}_{\mathscr{P}}^{2} \text { if } D \text { is ramified at } \mathscr{P} .
\end{array}\right.
$$

It is well known that the finitely generated two-sided submodules of $D$ (other than (0)) form an abelian group under ideal multiplication, which is freely generated by the $\Re_{\odot}$ 's.

By hypothesis, $T \theta=O T$ is a two-sided ideal in $\theta$. Therefore, we can write

$$
T \theta=\prod_{\mathscr{P}} \mathfrak{T}_{\Phi}^{n_{\Phi}}
$$

uniquely with $n_{\mathscr{P}} \geqslant 0$ for all $\mathscr{P}$.

If $\mathscr{P}$ is a prime of $K$, then $\overline{\mathcal{P}}=\mathscr{P}$, so that $\overline{\pi_{\mathscr{P}}}$ is a maximal ideal

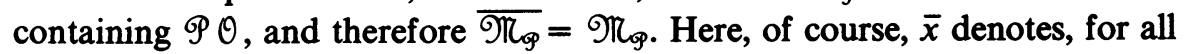
$x$, the image of $x$ under the canonical involution of $D$ over $K$. It follows that $\overline{\mathscr{B}}=\mathscr{B}$ for any two-sided $\mathcal{\theta}$-module $P$. Let $P=\left\{\mathscr{P}_{1}, \ldots, \mathscr{P}_{r}\right\}$. Taking reduced norms, we now see that

$$
\nu(T) \mathcal{O}_{K}=\prod_{\mathcal{P} \in P} \mathcal{P} n_{\mathscr{S}} . \prod_{\mathcal{P} \notin P} \mathcal{P}^{2 n_{\mathscr{\Phi}}} .
$$

Since the primes in $P$ generate the ideal class group of $K$, we can find an element $\xi \in K^{x}$, and integers $m_{1}, \ldots, m_{r}$ such that

$$
\prod_{\mathscr{P} \notin P} \mathcal{P} n_{\mathscr{P}}=\eta^{-1} \prod_{i=1}^{r} \mathcal{P P}_{i}^{m_{i}}
$$

Then we have

$$
\nu(\eta T) \mathcal{\Theta}_{K}=\prod_{i=1}^{r} \mathcal{P}_{i}^{n_{\Phi_{i}}+2 m_{i}}
$$

It is obvious that $T$ and $\eta T$ induce the same automorphism on $\Gamma$, that $\eta T \Theta=\theta \eta T$, and that $K(\sqrt{-\nu(T)})=K(\sqrt{-\nu(\eta T)})$. Therefore, replacing $T$ by $\eta T$ if necessary, we may assume without loss of generality that for $\mathscr{\rho} \notin P, n_{\mathscr{\rho}}=0$.

Since $\delta^{2}=-\nu(T)$, it follows that for $\mathscr{P} \notin P, \delta$ is a unit in $\theta_{\mathscr{\rho}}$, so that $\delta \theta_{\mathscr{P}}=\theta_{\mathscr{\rho}} \delta=\theta_{\mathscr{\rho}}$. For $\mathscr{\rho} \in P, D_{\mathscr{\rho}}$ is a division algebra, so $\delta \theta_{\mathscr{\rho}}=\theta_{\mathscr{\rho}} \delta$ automatically.

Therefore $\delta \theta=\theta \delta$. Since $\delta^{2}=-\nu(T)$, we have

$$
\begin{aligned}
(\delta \theta)^{2} & =\delta \theta \delta \theta=\delta^{2} \theta=\nu(T) \theta \\
& =T \bar{T} \theta=T \theta \bar{T} \theta=(T \theta)(\overline{T \theta})=(T \theta)^{2},
\end{aligned}
$$


so that $\delta \theta=T \theta$ and so $T \delta^{-1} \in \theta^{x}$. Therefore, $T \beta=T \delta c^{-2}=$ $T \delta(-\nu(T))^{-1}=T \delta \delta^{-2}=T \delta^{-1} \in \mathcal{O}^{x}$, so that

$$
\begin{aligned}
A(\mathfrak{U}) & =T \mathfrak{A} \beta=T \mathfrak{A} T^{-1} T \beta=T \vartheta \mathfrak{U} \Theta T^{-1} T \beta \\
& =(T \vartheta) \mathfrak{U}\left(T^{-1} \vartheta\right) T \beta=\mathfrak{A} T \beta=\mathfrak{A} .
\end{aligned}
$$

Proof of (4). It follows immediately from the result of $\S 6$ that $A_{0} \circ \varphi_{0}(x)$ $=\varphi_{0}\left(\tilde{\sigma}_{0} x\right) \circ A_{0}$. Since $B$ commutes with the elements of $\rho\left(D_{\mathrm{R}}\right)$, and since $\varphi_{0}(x) \in \rho\left(D_{\mathbf{R}}\right)$ for all $x$, we have

$$
\begin{aligned}
A \circ \varphi_{0}(x) & =B \circ A_{0} \circ \varphi_{0}(x)=B \circ \varphi_{0}\left(\tilde{\sigma}_{0} x\right) \circ A_{0} \\
& =-\varphi_{0}\left(\tilde{\sigma}_{0} x\right) \circ B \circ A_{0}=-\varphi_{0}\left(\tilde{\sigma}_{0} x\right) A,
\end{aligned}
$$

as required. Q.E.D.

9. In this section, we will describe the endomorphism ring of $V$. Let $\alpha \in \operatorname{End}(V)$, and let $\tilde{\alpha}$ be a holomorphic mapping of $X \times W$ onto itself which induces $\alpha$ on $U$. We can take $\tilde{\alpha}$ to be of the form $(x, u) \mapsto(x, \mathbb{Q}(u))$, where $\mathcal{Q}$ is a linear operator on $W$. It follows that $\mathbb{Q}$ must commute with the elements of $\rho(\Gamma)$ and therefore $\mathcal{Q}$ is of the form $u \mapsto u \cdot a$, with $a \in D_{\mathbf{R}}$. Since $\mathbb{Q}$ must preserve $\mathfrak{A}, a \in D$ and belongs to the right order $\theta^{\prime}$ of $\mathfrak{A}$. Conversely, if $a \in \mathcal{O}^{\prime}$, then one can show, using a proof similar to the proof of Theorem 2 , that $(x, u) \rightarrow(x, u a)$ induces an endomorphism of $V$. We omit the details. For a different approach, see Shimura [2].

10. Let $\sigma_{0}, \tilde{\sigma}_{0}, \psi, V$, and in particular $\mathfrak{A}$, be as in $\S 7$ of this chapter. Then $\mathfrak{U}=\boldsymbol{\theta}$, and so the right order of $\mathfrak{A}$ is $\theta$. In this way, we have $\operatorname{End}(V) \cong \theta$. Suppose that $\psi(\theta)=\theta$.

Let $\tilde{C}$ be the complex conjugation of $X \times W$ given by $\tilde{C}(x, u)=$ $\left(\tilde{\sigma}_{0} x, \psi(u)\right.$ ), and let $C$ be the complex conjugation induced on $V$ by $\tilde{C}$. Let $G=\{1, \psi\}$. We can view $\theta$ as a $G$-module in two different ways, as follows. For $\alpha \in \operatorname{End}(V), C \circ \alpha \circ C$ also belongs to $\operatorname{End}(V)$. Thus, letting $G$ act on $\operatorname{End}(V)$, by conjugation, via $\{1, C\}, \operatorname{End}(V)$ becomes a $G$-module.

Using the identification of $\operatorname{End}(V)$ with $\theta, \theta$ becomes a $G$-module, which we call $M_{1}$.

On the other hand, by hypothesis, $\psi(\theta)=\theta$, so that by letting $G$ act on $\theta$ via $\{1, \psi\}, \Theta$ becomes a $G$-module which we call $M_{2}$.

We will prove that $M_{1}=M_{2}$, in other words, that these two $G$-actions are the same.

Let $\alpha \in \theta$. Then $\alpha$ acts on $X \times W$ by the rule $(x, u) \alpha=(x, u \alpha)$. Call that mapping $\tilde{\alpha}$. The endomorphism $C \circ \alpha \circ C$ is induced on $V$ by the mapping $\tilde{C} \circ \tilde{\alpha} \circ \tilde{C}: X \times W \rightarrow X \times W$ given by $\tilde{C} \circ \tilde{\alpha} \circ \tilde{C}(x, u)=\tilde{C} \circ \tilde{\alpha}\left(\tilde{\sigma}_{0} x, \psi(u)\right)$ $=\tilde{C}\left(\tilde{\sigma}_{0} x, \psi(u) \alpha\right)=(x, u \psi(\alpha))$. Thus $C \circ \alpha \circ C$ is the endomorphism corresponding to $\psi(\alpha)$, which proves our assertion. 
11. By the results of Chapter III, $\S 7$, we can identify the set of $\operatorname{Aut}(V)$ conjugacy classes in $\omega\left(\sigma_{0}\right)$ with the cohomology set $H^{1}(G, \operatorname{Aut}(V))$. By the results of $\S 10$, we can identify this set with $H^{1}\left(\{1, \psi\}, \theta^{x}\right)$. Now suppose that $\psi$ is inner. Let $T \in \mathcal{O}$ such that $\psi(x)=T x T^{-1}$ for all $x \in D$. Since $\psi^{2}=1$, $T^{2} \in K$, and since $\psi \neq 1, T \notin K$. Therefore $\bar{T}=-T$, so $T^{2}=-\nu(T)$. By the results of Chapter III, $\S 6$, we can identify $H^{1}\left(\{1, \psi\}, \theta^{x}\right)$ with the set of $\theta^{x}$-conjugacy classes of elements $\delta \in \theta$ such that $\delta^{2}=-\nu(T)$. This cohomology set was computed in [4], [6].

12. We continue with the assumptions and notations of $\$ 10$. By the results of that section, the action of $\{1, \psi\}$ on $\theta$ via $\{1, c\}$ coincides with the usual action of $\{1, \psi\}$ on $\theta$.

On the other hand, the automorphism induced by $\tilde{\sigma}_{0}$ on $\Gamma$ coincides with $\psi$. Therefore, we can say unambiguously that the composition $\Gamma \hookrightarrow \theta^{x}$ $\stackrel{\sim}{\rightarrow} \operatorname{Aut}(V)$ is a morphism of $G$-modules.

By Chapter III, §2, there is an induced mapping $H^{1}(G, \Gamma) \rightarrow$ $H^{1}(G, \operatorname{Aut}(V))$. By the results of Chapter III, $\S 5$, we can identify $H^{1}(G, \Gamma)$ with the set of path components of the fixpoint set of $\sigma_{0}$.

It follows now that every component of the fixed point set of $\sigma_{0}$ determines an $\operatorname{Aut}(V)$-conjugacy class of liftings of $\sigma_{0}$ to $V$.

13. If $N$ is a positive integer, we denote by $\Gamma_{N}$ the group of all $\gamma \in \theta^{1}$ such that $\gamma-1 \in N \Theta$. For sufficiently large $N, \Gamma_{N}$ is torsion-free, and for all $N$, $\Gamma_{N}$ has finite index in $\theta^{1}$.

In this section, we will compute $H^{1}\left(\Gamma_{N}, \theta\right)^{\text {tors }}$. Our result is that $H^{1}\left(\Gamma_{N}, \theta\right)^{\text {tors }}$ is isomorphic to $\theta / N \theta$.

For every prime $\mathcal{P}$ of $K$, denote by $K_{\mathscr{P}}$ the completion of $K$ at $\mathscr{P}$ and by $\Theta_{\mathscr{\rho}}$ the closure of $\mathcal{\theta}$ in $D_{\mathscr{P}}=D \otimes_{K} K_{\mathscr{\rho}}$. Let $\Theta_{\mathscr{P}}^{x}$ denote the group of units of $\theta_{\mathscr{\Phi}}$, and write $\Gamma_{N}^{\Phi}$ for the subgroup of $\theta_{\mathscr{\Phi}}^{x}$ consisting of all $\gamma \in \theta_{\Phi}^{x}$ whose reduced norm is 1 , and such that $\gamma-1 \in N \theta_{\Phi}$. By the approximation theorem, $\Gamma_{N}$ is dense in $\Gamma_{N}^{\mathscr{P}}$ for every $\mathscr{P}$.

Let $n_{\mathscr{P}}=\operatorname{ord}_{\mathscr{P}} N$ for every $\mathcal{P}$. Then $\Gamma_{N}$ and $\Gamma_{N}^{\mathscr{P}}$ have the same image in $\theta_{\mathscr{P}} / \mathcal{P}^{n+l} \theta_{\mathscr{P}}$ for every $l>0$, namely they both map onto

$$
\left\{x \in \theta_{\mathscr{\Phi}} / \mathscr{\rho} n_{\mathscr{P}}+l \theta_{\mathscr{P}} \mid \nu(x)=1 \text { and } x-1 \in \mathcal{P}^{n} \hat{\theta}_{\mathscr{P}} / \mathscr{\rho} n_{\mathscr{P}}+l \theta_{\mathscr{P}}\right\} \text {. }
$$

Here, of course, $\nu$ denotes the mapping $\theta_{\mathscr{P}} / \mathcal{P}^{n_{\Phi}+l} \theta_{\mathscr{P}} \rightarrow \theta_{K_{\Phi}} / \mathcal{P}^{n_{\Phi}+l} \theta_{K_{\mathscr{\Phi}}}$ induced by the reduced norm mapping $D \rightarrow K$.

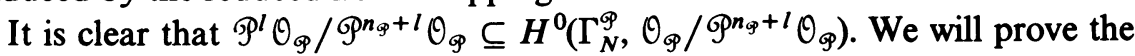
inclusion is actually an equality.

Case I. $D$ is ramified at $\mathscr{P}$. Then $D_{\mathscr{P}}$ is a division algebra over $K_{\mathscr{P}}$ and $\Theta_{\mathscr{P}}$ is a valuation ring with maximal ideal $\mathscr{\rho}$. Therefore $\theta_{\mathscr{P}} / \mathcal{P}^{n_{\mathscr{P}}+l} \theta_{\mathscr{P}}$ is a valuation ring whose maximal ideal is $\mathcal{P} \theta_{\mathscr{P}} / \mathscr{P}^{n_{\mathscr{P}}+l} \Theta_{\mathscr{P}}$. Let $x \in H^{0}\left(\Gamma_{N}^{\mathscr{P}}, \Theta_{\mathscr{P}} / \mathscr{P}^{n_{\mathcal{P}}+l} \Theta_{\mathscr{P}}\right)$. 
Let $\gamma \in \Gamma_{N}^{\Phi}$ be an element such that its image in $\theta_{\Phi} / \rho^{\rho n_{\Phi}+l} \theta_{\mathscr{\Phi}}$ does not

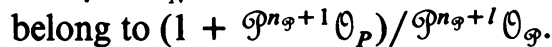

This is possible in view of our determination of the image of $\Gamma_{N}^{\mathscr{P}}$ in $\theta_{\mathscr{\Phi}} / \Phi^{n_{\Phi}+l} \theta_{\mathscr{\Phi}}$. We have ord $(\bar{\gamma}-1) \leqslant n_{\Phi}$.

Since $x=\bar{\gamma} x=x+(\bar{\gamma}-1) x$, we have $(\bar{\gamma}-1) x=0$. Therefore $\operatorname{ord}_{\mathscr{P}} x>$ $\left(n_{\mathscr{P}}+l\right)-\operatorname{ord}_{\mathscr{\Phi}}(\bar{\gamma}-1) \geqslant l$, which proves that $x \in \mathscr{P}^{l} \theta_{\Phi} / \mathscr{P}^{n_{\mathscr{S}}+l} \theta_{\mathscr{\Phi}}$. Therefore $\mathscr{P}^{l} \theta_{\mathscr{P}} \mid \mathscr{P}^{n_{\mathscr{P}}+l} \theta_{\mathscr{P}}=H^{0}\left(\Gamma_{N}^{\mathscr{S}}, \theta_{\mathscr{\Phi}} / \mathcal{P}^{n_{\mathscr{P}}+l} \theta_{\mathscr{\Phi}}\right)$.

Case II. $D$ is unramified at $\mathscr{P}$. Then $D_{\Phi} \cong M_{2}\left(K_{\Phi}\right)$ and $\theta_{\mathscr{\Phi}} \cong M_{2}\left(\theta_{K_{\Phi}}\right)$.

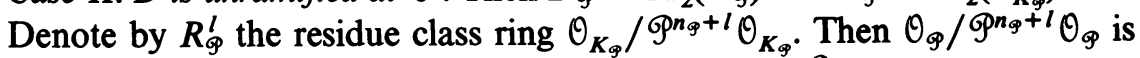
isomorphic to $M_{2}\left(R_{\Phi}^{l}\right)$ in such a way that the image of $\Gamma_{N}^{\Phi}$ corresponds to

$$
\left\{x \in M_{2}\left(R_{\Phi}^{l}\right) \mid \operatorname{det} x=1 \text { and } x \equiv\left(\begin{array}{ll}
1 & 0 \\
0 & 1
\end{array}\right)\left(\bmod \mathcal{P}^{n_{\Phi}} M_{2}\left(R_{\odot}^{l}\right)\right)\right\} \text {. }
$$

Let $\pi \in \mathcal{O}_{K_{\mathscr{q}}}$ be a prime element, and denote by $\bar{\pi}$ its image in $\boldsymbol{R}_{\Phi}^{l}$.

Then

$$
\left(\begin{array}{ll}
1 & \bar{\pi}^{n_{\mathcal{S}}} \\
0 & 1
\end{array}\right) \text { and }\left(\begin{array}{ll}
1 & 0 \\
\bar{\pi}^{n_{\mathcal{S}}} & 1
\end{array}\right)
$$

both belong to the image of $\Gamma_{N}^{\Phi}$ in $M_{2}\left(R_{\odot)}^{l}\right)$.

Let

$$
\left(\begin{array}{ll}
a & b \\
c & d
\end{array}\right) \in H^{0}\left(\Gamma_{N}^{\mathscr{P}}, \theta_{\mathscr{\Phi}} / \mathcal{P}^{n}+l \theta_{\mathscr{P}}\right)
$$

Then

$$
\left(\begin{array}{ll}
a & b \\
c & d
\end{array}\right)=\left(\begin{array}{ll}
1 & \bar{\pi}^{n_{\oplus}} \\
0 & 1
\end{array}\right)\left(\begin{array}{ll}
a & b \\
c & d
\end{array}\right)=\left(\begin{array}{cc}
a+\bar{\pi}^{n_{\Phi_{c}}} & b+\bar{\pi}^{n_{\Phi_{d}}} \\
c & d
\end{array}\right)
$$

and

$$
\left(\begin{array}{ll}
a & b \\
c & d
\end{array}\right)=\left(\begin{array}{cc}
1 & 0 \\
\bar{\pi}^{n_{\Phi_{1}}} & 1
\end{array}\right)\left(\begin{array}{ll}
a & b \\
c & d
\end{array}\right)=\left(\begin{array}{cc}
a & b \\
\bar{\pi}^{n_{\Phi_{a+c}}} & \bar{\pi}^{n_{\Phi_{b+d}}}
\end{array}\right)
$$

so that $\bar{\pi}^{\Phi_{P_{a}}}+\bar{\pi}^{n_{\Phi_{b}}}=\bar{\pi}^{n_{\Phi_{c}}}=\bar{\pi}^{n_{\Phi_{d}}}=0$. Therefore, $a, b, c, d \in \mathcal{P}^{l} R_{\Phi}^{l}$, so that

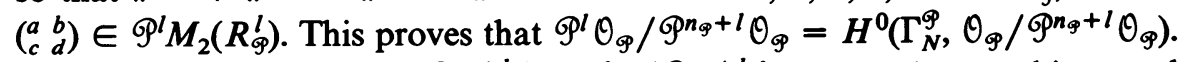

Since the inclusion $\theta / \Phi^{n_{\Phi}+l} \theta \hookrightarrow \theta_{\Phi} / \Phi^{n^{n}+l} \theta_{\Phi}$ is an isomrophism and since $\Gamma_{N}$ and $\Gamma_{N}^{\Phi}$ have the same image in $\theta_{\Phi} / \Phi^{\rho} n^{+}+l \theta_{\Phi}$, we conclude that

$$
\mathscr{P}^{l} \theta / \mathscr{P}^{n_{\mathcal{Q}}+l} \boldsymbol{\theta}=H^{0}\left(\Gamma_{N}, \theta / \mathscr{P}^{n_{\mathcal{P}}+l} \theta\right) \text {. }
$$

As we remarked in Chapter $I, \$ 8$, for any positive integer $M$, $H^{0}\left(\Gamma_{N}, \theta / M N \theta\right)$ is naturally isomorphic to the $M N$-torsion in $H^{1}\left(\Gamma_{N}, \theta\right)$. Moreover, we observed that $H^{1}\left(\Gamma_{N}, \theta\right)^{\text {tors }}$ is a finite group. Therefore, for suitable $M$, we have $H^{0}\left(\Gamma_{N}, \theta / M N \theta\right) \cong H^{1}\left(\Gamma_{N}, \theta\right)^{\text {tors }}$.

For every prime $\mathcal{P}$ of $K$, let $l_{\mathscr{\rho}}=\operatorname{ord}_{\mathscr{P}} M$. Then $\theta / M N \theta$ $\simeq_{\Gamma_{N}} \theta / \rho \rho^{n_{g}+l_{\rho}} \theta$, so that by what we have proved above, we have 


$$
\begin{aligned}
H^{0}\left(\Gamma_{N}, \theta / M N \theta\right) & \cong \bigoplus_{\mathscr{P}} H^{0}\left(\Gamma_{N}, \theta / \mathscr{P}^{n_{\mathscr{P}}+l_{\mathscr{P}}} \theta\right) \\
& \cong \bigoplus_{\mathscr{P}} \mathscr{P}^{l_{\mathscr{\Phi}}} \theta / \mathscr{P}^{n_{\mathscr{P}}+l_{\mathscr{\Phi}}} \theta \cong M \theta / M N \theta \cong \theta / N \theta .
\end{aligned}
$$

Therefore, $H^{1}\left(\Gamma_{N}, \theta\right)^{\text {tors }} \cong \theta / N \theta$.

\section{BIBLIOGRAPHY}

1. M. Kuga, Fibre varieties over a symmetric space whose fibres are abelian varieties, Lecture Notes, Univ. of Chicago, Chicago, Ill., 1964.

2. G. Shimura, On analytic families of polarized abelian varieties and automorphic functions, Ann. of Math. 78 (1963).

3. Introduction to the arithmetic theory of automorphic functions, Publ. Math. Soc. Japan 11 (1971).

4. S. Kudla, On the R-forms of certain algebraic varieties, Bull. Amer. Math. Soc. 81 (1975), 471-473.

5. H. Jaffee, Real forms of hermitian symmetric spaces, Bull. Amer. Math. Soc. 81 (1975), 456-458.

6. Shimizu, On the zeta function of a quaternion algebra, Ann. of Math. 196.

7. G. Shimura, On the real points of an arithmetic quotient of a bounded symmetric domain, Math. Ann. 215 (1975), 135-164. Also, On abelian varieties with complex multiplication, Proc. London Math. Soc. 34, 65-86. (Especially, pp. 79-82.)

8. K.-Y. Shih, Antiholomorphic automorphisms of arithmetic automorphic function fields, Ann. of Math. 103 (1976), 81-102.

Department of Mathematics, State University of New York at Stony Brook, Stony BROOK, NEW YORK 11794 